\title{
1 Asymmetric effects of acute stress on cost and benefit learning
}

2 Stella Voulgaropoulou ${ }^{1 *}$, Fasya Fauzani ${ }^{1}$, Janine Pfirrmann ${ }^{1}$, Claudia Vingerhoets ${ }^{1,2}$, Thérèse

3 van Amelsvoort $^{1}$, Dennis Hernaus ${ }^{1}$

$5 \quad{ }^{1}$ Department of Psychiatry \& Neuropsychology, Maastricht University, Minderbroedersberg

6 4-6, 6211 LK Maastricht, The Netherlands

$7 \quad{ }^{2}$ Department of Radiology \& Nuclear Medicine, Amsterdam University Medical Centre,

8 Location AMC, Meibergdreef 5, 1105 AZ Amsterdam, The Netherlands.

10 Abstract

11 Stressful events trigger a complex physiological reaction - the fight-or-flight response - that

12 can hamper flexible decision-making. Inspired by key neural and peripheral characteristics of

13 the fight-or-flight response, here we ask whether acute stress changes how humans learn

14 about costs and benefits. Participants were randomly exposed to an acute stress or no-stress

15 control condition after which they completed a cost-benefit reinforcement learning task.

16 Acute stress improved learning to maximize benefits (monetary rewards) relative to

17 minimising energy expenditure (grip force). Using computational modelling, we demonstrate

18 that costs and benefits can exert asymmetric effects on decisions when prediction errors that

19 convey information about the reward value and cost of actions receive inappropriate

20 importance; a process associated with distinct alterations in pupil size fluctuations. These

21 results provide new insights into learning strategies under acute stress - which, depending on

22 the context, may be maladaptive or beneficial - and candidate neuromodulatory mechanisms

23 that could underlie such behaviour. 


\section{Introduction}

25 Stress is ubiquitous in everyday life. From recurrent, brief, events (a work meeting, moving to a new house) to major life events (armed combat, pandemic, financial crisis), humans are continuously exposed to challenges in their daily environment. The immediate central and peripheral physiological cascade triggered by such events, collectively termed the fight-orflight (or acute stress) response (Cannon, 1915), serves an allostatic role that enables organisms to adequately respond to environmental demands (de Kloet, Joëls, \& Holsboer, 2005). Although beneficial for survival, this allostatic process comes at a cost: stress-induced redistributions of neural resources - e.g., towards vigilance or threat detection - may hamper the deployment of strategies that support adaptive and optimal decision-making (Hermans, Henckens, Joëls, \& Fernández, 2014). and negative outcomes of previous actions, also known as reinforcement learning (Niv, 2009). Considerable evidence now suggests that acute stress impairs aspects of reinforcement learning (Carvalheiro, Conceição, Mesquita, \& Seara-Cardoso, 2020; de Berker et al., 2016;

39 Raio, Hartley, Orederu, Li, \& Phelps, 2017). Acute stress, among others, modulates the impact of positive outcomes on future decisions - both positively and negatively - (Berghorst,

41 Bogdan, Frank, \& Pizzagalli, 2013; Carvalheiro et al., 2020; Lighthall, Gorlick, Schoeke, Frank, \& Mather, 2013; Petzold, Plessow, Goschke, \& Kirschbaum, 2010), likely driven by changes in reward sensitivity and the signalling of reward prediction errors (RPEs)

44 (Berghorst et al., 2013; Carvalheiro et al., 2020; Huys, Pizzagalli, Bogdan, \& Dayan, 2013); putatively dopaminergic teaching signals that represent the mismatch between actual and expected outcomes, which are used to flexibly adjust behaviour (Niv, 2009; Rescorla, 1972).

47 Alterations in the influence of RPEs on future decisions play a key role in the development of 
motivational impairments, which are frequently observed in behavioural disorders associated

with repeated and/or prolonged stress exposure (Huys et al., 2013).

decisions primarily involves changes in how reward value influences action may be

oversimplified. Decisions are not only motivated by appetitive properties; they equally

depend on the - cognitive (e.g., mental effort) or physical (e.g., energy) - cost associated

with actions (Hauser, Eldar, \& Dolan, 2017; Pessiglione, Vinckier, Bouret, Daunizeau, \& Le

Bouc, 2017; Schmidt, Lebreton, Cléry-Melin, Daunizeau, \& Pessiglione, 2012). Expectations

about action costs are also updated according to a prediction error rule (Skvortsova, Degos,

Welter, Vidailhet, \& Pessiglione, 2017; Skvortsova, Palminteri, \& Pessiglione, 2014)

(henceforth "effort" prediction errors; EPEs), which due to the aversive and resource-

consuming nature of effort, optimal learners should utilize to minimize effort expenditure.

When decisions involve a potential cost and benefit, the former is subtracted from the latter to compute a "net" or subjective decision value (i.e., effort-discounted reward value) (KleinFlügge, Kennerley, Friston, \& Bestmann, 2016; Skvortsova et al., 2017; Skvortsova et al., 2014). Notably, stress exposure impairs cost-benefit decisions in rodents when learning is not explicitly required (Friedman et al., 2017; Shafiei, Gray, Viau, \& Floresco, 2012). Moreover, in a reinforcement learning context, acute stress blocks the flexible updating of aversive value (Raio et al., 2017), an inherent property of costly actions. These results suggest that decisions during acute stress may involve a complex shift in reinforcement learning strategies that serve to balance the cost versus benefits of decisions; a hypothesis that hitherto has remained unexplored.

Although computationally similar in nature, distinct neural correlates of RPEs (e.g., striatal subdivisions, ventromedial prefrontal cortex [vmPFC]) and EPEs (e.g., parietal cortex, insula, dorsomedial PFC) can be observed in cost-benefit reinforcement learning 
paradigms (Hauser et al., 2017; Skvortsova et al., 2014). The ascending dopaminergic (e.g.,

74 RPEs, action cost, reward value) (Schultz, Dayan, \& Montague, 1997; Skvortsova et al.,

2017; Yohn et al., 2016), noradrenergic (e.g., mobilizing energy) (Pessiglione et al., 2017;

Varazzani, San-Galli, Gilardeau, \& Bouret, 2015) and serotonergic (e.g., aversive value,

overcoming action costs) (H. E. den Ouden et al., 2015; Meyniel et al., 2016)

neuromodulatory systems, moreover, encode partly dissociable aspects of goal-directed

actions that involve learning about costs and benefits, which together support optimal

decision-making. These observations are noteworthy because the initial fight-or-flight

response triggers a large-scale reorganization of brain networks that is driven by alterations in

the firing mode of midbrain dopaminergic ventral tegmental area and noradrenergic locus coeruleus neurons (Arnsten, 2015; Hermans et al., 2014); neurons that signal prediction errors (Steinberg et al., 2013) and that are also responsive to reward value, action cost and energy expenditure (Del Arco, Park, \& Moghaddam, 2020; Varazzani et al., 2015). Thus, catecholaminergic mechanisms that are recruited by the fight-or-flight response may differentially impact cost and benefit reinforcement learning, resulting in a potential scenario in which costs and benefits exert asymmetric influences on decisions. cognitive strategies, including reinforcement learning. The peripheral counterpart of the acute stress response, however, mobilizes the energy (i.e., adrenaline-mediated glucose release (de Kloet et al., 2005; Russell \& Lightman, 2019)) that is required to exert effortful actions aimed at preserving homeostasis (Cannon, 1915). Therefore, decision-making and learning policies regarding physical costs may be especially susceptible to stress: both via computational (neural) mechanisms that support learning about and representation of action cost, as well as peripheral mechanisms that co-determine the amount of available energy that can be directed towards effortful actions. Indeed, preliminary evidence suggests that acute 
stress alters the willingness to exert physical effort for rewards (Bryce \& Floresco, 2016) and

Delplanque, \& Sander, 2015).

How acute stress impacts reinforcement learning involving costs and benefits has not

102 been investigated to date in humans. Based on the above considerations, we expect that

103 computationally frugal learning strategies, in concert with increased energy availability,

104 during acute stress should asymmetrically impact cost versus benefit learning. Using an acute

105 stress-induction paradigm, a cost-benefit learning paradigm and computational model of cost-

106 benefit reinforcement learning (Skvortsova et al., 2017; Skvortsova et al., 2014), we

107 demonstrate that acute stress asymmetrically prioritizes reward (maximization) learning over

108 physical effort (minimization) learning. Better benefit versus cost learning results from a

109 stress-induced change in the influence of RPEs versus EPEs on future decisions, and is

110 associated with altered pupil encoding of RPEs, EPEs, and subjective decision value. These

111 results reveal how neural and peripheral mechanisms that support the fight-or-flight response

112 may facilitate a shift in reinforcement learning strategies that confers strategic benefits during

113 acutely stressful situations (e.g., ignoring high action costs to achieve a desirable outcome),

114 yet might also give rise to maladaptive behaviour (e.g., stress-induced relapse in substance

115 users). 


\section{Results}

\section{Experiment design}

118 Healthy human participants were randomly assigned to the acute stress (19 males/21 females;

119 age $\mathrm{M}=23.48, \mathrm{SD}=3.94$ ) or no-stress control condition (18 males/22 females; age $\mathrm{M}=23.80$,

$120 \mathrm{SD}=4.23$ ) of the Maastricht Acute Stress Task (MAST) (Smeets et al., 2012), a validated

121 psychological and physical stress-induction paradigm (see Materials and Methods).

122 Immediately post-MAST and within the confines of the acute stress response (Hermans et al.,

123 2014), all participants completed a $\sim 40$ minute probabilistic cost-benefit reinforcement

124 learning paradigm, adapted from Skvortsova et al. (Skvortsova et al., 2017; Skvortsova et al.,

125 2014), in which they learned to select stimuli with high reward value (20 Eurocents) and

126 avoid stimuli with high action cost (exerting grip force above a pre-calibrated individual

127 threshold of $50 \%$ maximum voluntary contraction for $3000 \mathrm{~ms}$ ), followed by a surprise test

128 phase. A detailed overview of the paradigm is provided in Figure 1 and the Materials and

129 Methods. Pupil size was continuously recorded while participants performed the task (see

130 Materials and Methods). 


\section{Figure 1}

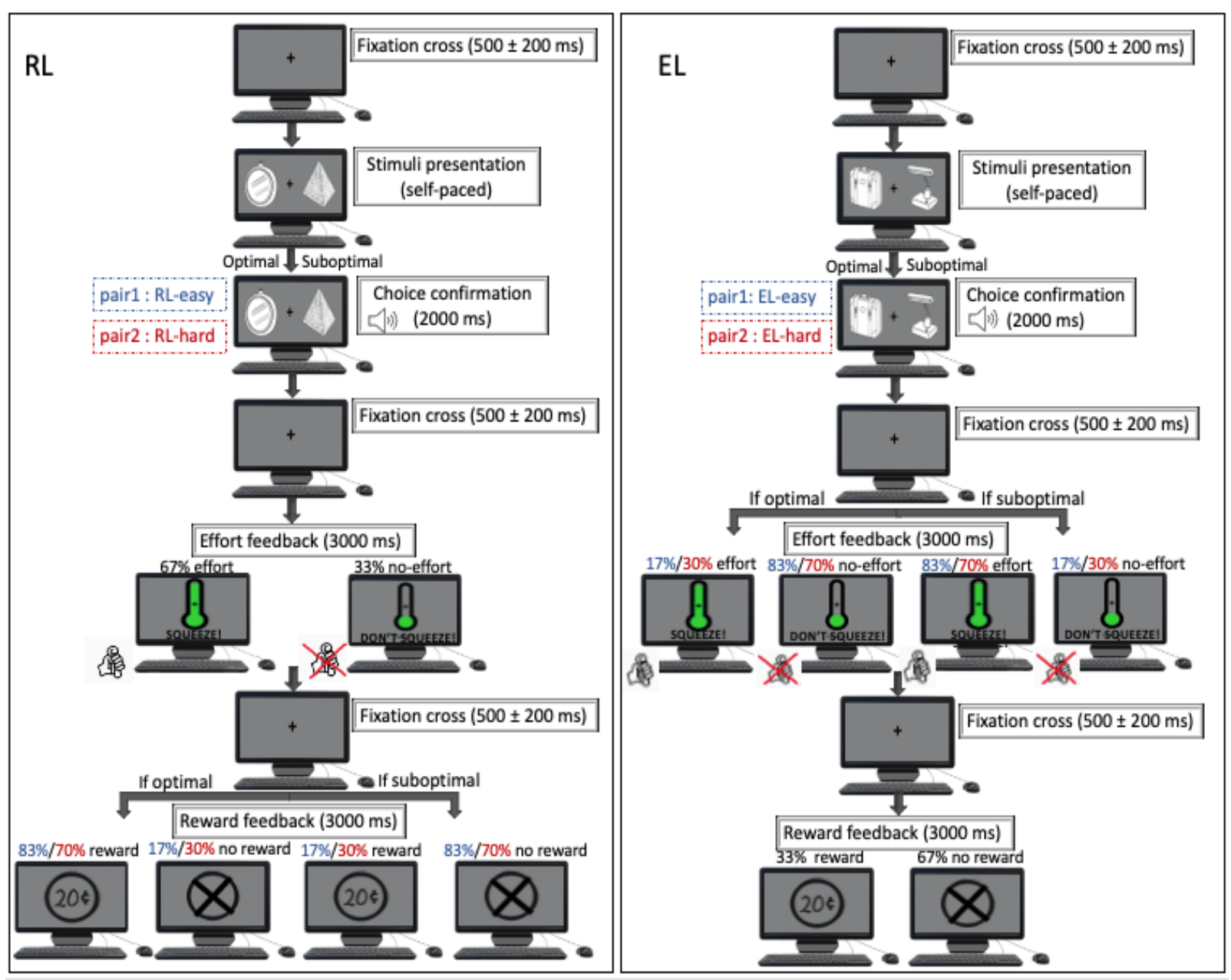

Reward maximisation/action cost minimization reinforcement learning task.

134 Visual depiction of the learning phase. Participants were presented with four distinct stimulus

135 pairs, and all stimuli were associated with a predetermined chance of a $€ 0.20$ monetary

136 reward (versus no reward) and a chance of having to exert physical effort (grip force) using a

137 dynamometer (versus no grip force required). Stimulus-outcome probabilities were yoked in

138 such a way that, for a given pair, two stimuli only differed in the probability of earning a

139 reward ("reward learning", RL, left) or the probability of having to exert effort ("effort

140 learning”, EL, right). That is, for RL (left)/EL (right) pairs, reward/effort outcomes were

141 choice-dependent, respectively (see "Reward feedback" for RL and "Effort feedback" for EL

142 for outcome contingencies). For RL pairs, effort outcomes were independent of choice and

143 fixed (see "Effort feedback" for RL), while for EL pairs, reward outcomes were independent 
144 of choice and fixed (see "Reward feedback" for EL). Percentages in blue and red refer to

145 outcomes for the Easy RL/EL and Hard RL/EL pair, respectively.

\section{Acute stress manipulation}

148 We first ascertained whether the acute stress manipulation was successful. Subjective stress,

149 physiological and neuroendocrine measurements are displayed in Figure 2. Acute stress and no-stress control groups did not differ on physiological, subjective stress, or neuroendocrine measurements pre-MAST (all $p$-values $>0.05$ ). We observed significant Condition-by-Time

152 interactions for subjective stress ratings [PANAS negative: $F(1,78)=52.66, p<0.001$,

$153 n^{2}=0.10$; PANAS positive: $\left.F(1,78)=9.82 p=0.002, n^{2}{ }_{G}=0.02\right]$ and physiological measures

154 [systolic blood pressure (SBP): $F(1,78)=15.50, p<0.001, n^{2}{ }_{G}=0.04$; heart rate: $F(1,78)=6.83$, $\left.p=0.011, n^{2}{ }_{G}=0.02\right]$. Simple main effect analyses revealed that only the acute stress group exhibited pre-to-post increases in negative affect [control pre-post: $t(39)=4.21, p<0.001$; stress pre-post: $t(39)=-6.17, p<0.001$; control-stress post-MAST: $t(55.1)=-5.78, p<0.001]$, and greater pre-to-post decreases in positive affect [control pre-post: $t(39)=4.09, p<0.001$; stress pre-post: $t(39)=6.45, p<0.001$; control-stress post-MAST: $t(72.8)=2.53, p=0.014]$ (Figure 2). Similarly, only the acute stress group exhibited stress-induced increases in SBP

161 [control pre-post: $t(39)=1.60, p<0.117$; stress pre-post: $t(39)=-3.66, p<0.001$; control-stress post-MAST: $t(69.1)=-3.27, p=0.002]$ and heart rate [control pre-post: $t(39)=1.21, p=0.234$; stress pre-post: $t(39)=-2.78, p=0.008$; control-stress post-MAST: $t(76.9)=-3.14, p=0.002]$

164 (Figure 2a-d).

An expected Condition-by-Time interaction was found for salivary cortisol (sCORT) responses $\left[F(5,390)=18.05, p<0.001, n^{2} G=0.04\right]$, with the acute stress group displaying

167 greater sCORT levels 10 min post-MAST and onwards (all $p$-values $<0.01$ ). We additionally 168 observed a main effect of Condition on sCORT area-under-the-curve with respect to increase: 
169 (AUCi) (Pruessner, Kirschbaum, Meinlschmid, \& Hellhammer, 2003) $(t(56.32)=-5.28$,

$170 p<0.001)$ and salivary alpha-amylase (sAA) AUCi $(t(67.45)=-2.50, p=0.015$; after excluding

171 one extreme outlier from the control group), suggesting greater sCORT and sAA levels in

172 response to acute stress (Figure 2e-g). These results confirm that the MAST robustly induced

173 stress on all levels of inquiry.

Figure 2

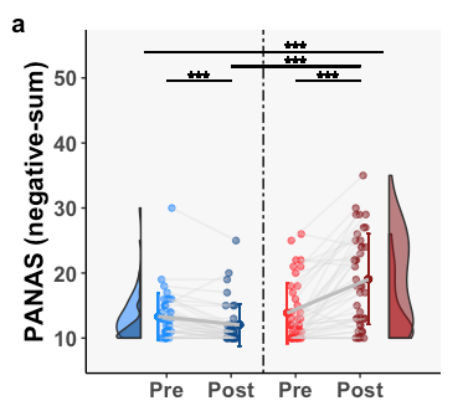

e

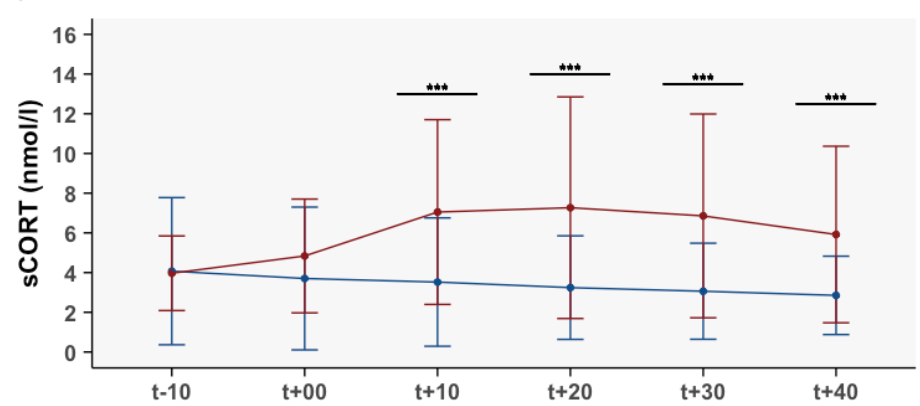

C
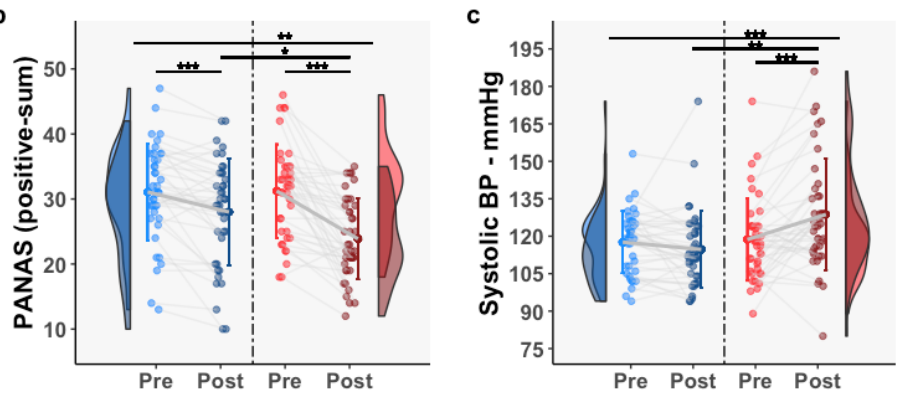

Condition/Timepoint $\rightarrow$ control-p

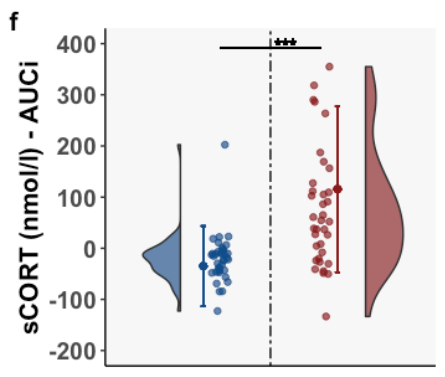

d
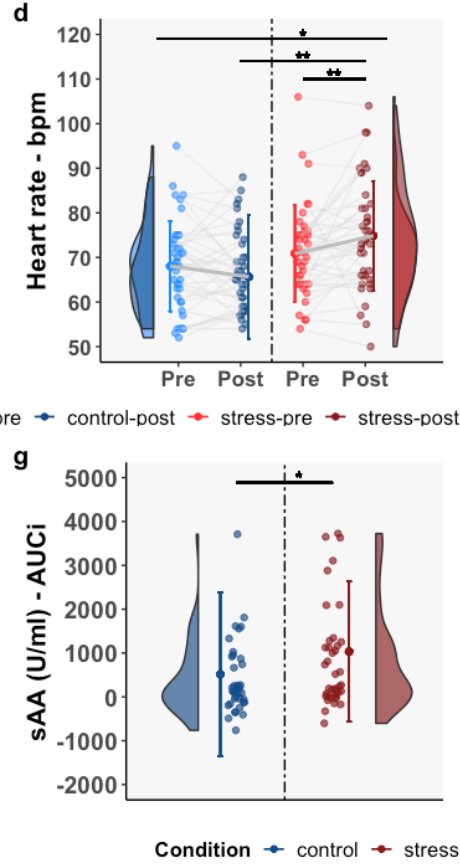

177 Neuroendocrine, physiological and subjective stress ratings.

178 PANAS negative (a) and positive (b) subscale sum scores, systolic blood pressure (mmHg:

179 millimetres of mercury; c) and heart rate (bpm: beats per minute; d) are displayed for no-

180 stress control (blue) and acute stress (red) groups separately for pre (light blue/red) and post

181 (dark blue/red) MAST time points. SCORT responses for both conditions across the 6

182 timepoints are displayed in panel $\mathbf{e}($ " $t+00$ " represents the first post-MAST measurement, and

183 the start of the reward maximization/action cost reinforcement learning paradigm; " $\mathrm{t}-10$ "

184 represent a baseline sample). Panel $\mathbf{f}$ and $\mathbf{g}$ show AUCi for sCORT (nmol/1: nanomoles per

185 litre) and sAA (U/mL: Units per millilitre) responses for both MAST conditions. Significant 
differences are denoted by asterisks $\left(*: p<0.05,{ }^{*}: p<0.01,{ }^{* * *}: p<0.001\right)$. In the upper simple main effects of Condition or Time.

\section{Participants use reinforcement learning to optimize decisions}

191 Next, we investigated whether participants in both conditions exhibited evidence of action cost as a function of increasing number of stimulus pair presentations (i.e., "time"). which participants could learn to accumulate frequent rewards while the probability of effort

197 (action cost) was kept constant (RL; selecting the stimulus more frequently associated with $€ 0.20)$ [control: $F(2,78)=10.16, p<0.001, n^{2}{ }_{G}=0.06$; stress: $F(2,78)=20.44, p<0.001, n^{2}{ }_{G}=$ probability of reward was kept constant (EL; selecting the stimulus more frequently associated with avoidance of physical energy expenditure) [control: $F(2,78)=12.35, p<0.001$, $n^{2}{ }_{G}=0.07$; stress: $\left.F(2,78)=9.76, p<0.001, n^{2}{ }_{G}=0.05\right]$. We additionally observed greater than chance-level performance $(\geq 0.5)$ on both trial types during the final part of the task (presentation 21-30; all $p$-values $<0.001$ Figure Supplement 1).

\section{Asymmetric cost-benefit reinforcement learning during acute stress}

207 After having observed evidence for reward (maximization) learning and action cost

208 (minimization) learning, we tested our key assumption; that acute stress would induce a

209 reprioritization in learning to maximize reward value versus learning to minimize action cost.

210 Crucially, we observed a significant Condition-by-Trial Type interaction $[F(1,78)=6.53$, 
$211 p=0.013, n^{2}{ }_{G}=0.039$ ] (Figure 3a) with pairwise comparisons indicating that the acute stress

212 group performed significantly better on RL than EL trials $[t(39)=5.40, p<0.001]$, while the

213 no-stress control group performed similarly on both trial types $[t(39)=1.01, p=0.320]$. A main

214 effect of Condition on RL-EL accuracy difference scores $[t(74.02)=-2.55, p=0.013]$ (Figure

$2153 \mathrm{~b}$ ) and one-sample t-tests revealed that RL-EL accuracy difference scores were significantly

216 greater than zero in the acute stress group but not in the no-stress controls. Simple main

217 effects of Condition on $\operatorname{RL}[t(65.9)=-1.75, p=0.085]$ and $\operatorname{EL}[t(77.5)=1.80, p=0.076]$

218 performance showed numerical trends for group differences that failed to reach significance.

219 When we included participants that still performed at chance level at the end of the

220 learning phase (see Participants) in the Condition-by-Trial Type interaction analysis, the

221 interaction remained significant $\left[F(1,91)=7.30, p=0.035, n^{2} G=0.04\right]$, with the acute stress

222 group displaying better RL vs. EL performance $(t(46)=5.83, p<0.001)$, while no-stress

223 controls performed similarly on both trial types $(t(45)=1.24, p=0.22)$. Participants in the acute

224 stress group outperformed participants in the no-stress control group on $\operatorname{RL}(t(91)=2.04$,

$225 p=0.04)$, but no simple main effect of Condition was observed for EL $(t(91)=-1.67, p=0.1)$.

226 These participants were excluded for all other analyses reported below.

The use of different reinforcement probabilities for each RL and EL pair (Figure 1)

allowed us to discern whether the observed pattern of results reflected a specific change in

cost (EL) versus benefit (RL) reinforcement learning, or a more general impairment in

230 reinforcement learning for more difficult stimulus-response associations. We found no

231 evidence for the latter scenario in Condition-by-Trial Type-by-Difficulty $[F(1,78)=1.05$,

$232 p=0.310]$ or Condition-by-Difficulty $[F(1,78)=0.36, p=0.549$; Figure Supplement 2]

233 interaction analyses.

234 When we investigated the use of win-stay (resampling a stimulus following a positive

235 outcome) and lose-shift (switching to the other stimulus following a negative outcome) 
strategies (Hanneke E. M. den Ouden et al., 2013), we observed no significant Condition-by-

237 Strategy interaction $\left[F(1,78)=2.99, p=0.087, n^{2}{ }_{G}=0.03\right]$. However, post hoc comparisons

238 revealed that participants in the acute stress condition $[t(39)=-3.73, p<0.001]$ but not those in

239 the no-stress control condition $[t(39)=-1.30, p=0.200]$, exhibited different win-stay compared

240 to lose-shift (difference) rates. Separate Condition (main effect) analyses indicated that acute

241 stress participants compared to no-stress controls were more likely to win-stay for rewards

242 (RL trials) than for avoidance of action cost (EL trials) $[t(78)=-2.28, p=0.025]$, but not for

243 lose-shifting for reward omissions (RL trials) compared to exerting effort (EL trials)

$244[t(77.7)=-0.23, p=0.820]$. Differences in win-stay rates for RL compared to EL trials (one-

245 sample t-test) were greater than zero for the acute stress $[t(39)=4.91, p<0.001]$ but not the no-

246 stress control group $[t(39)=1.68, p=0.101]$ (Figure $3 c$ ).

247 Taken together, our model-free results indicate that acute stress leads to a

248 reinforcement learning strategy that favours learning to maximise reward value over

249 minimisation of action cost, which based on analyses of win-stay/lose-shift rates, could be

250 attributed to increased sensitivity to positive reinforcement (i.e., reward delivery) compared

251 to negative reinforcement (i.e., avoidance of physical effort). 
a

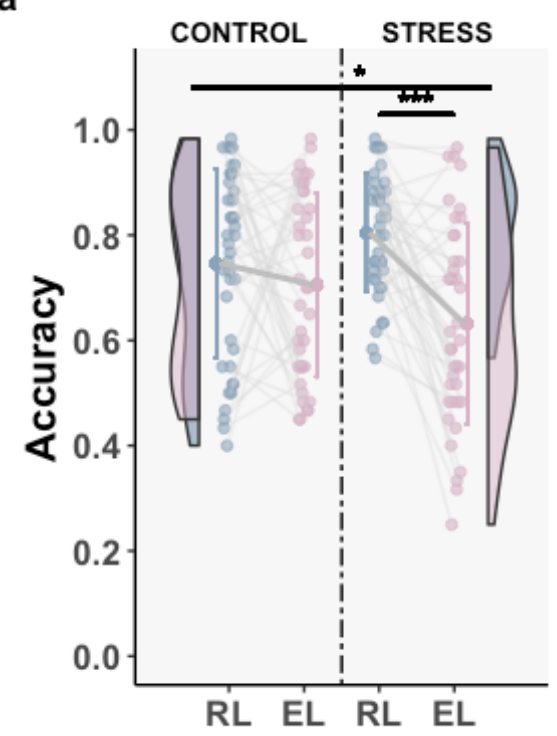

b

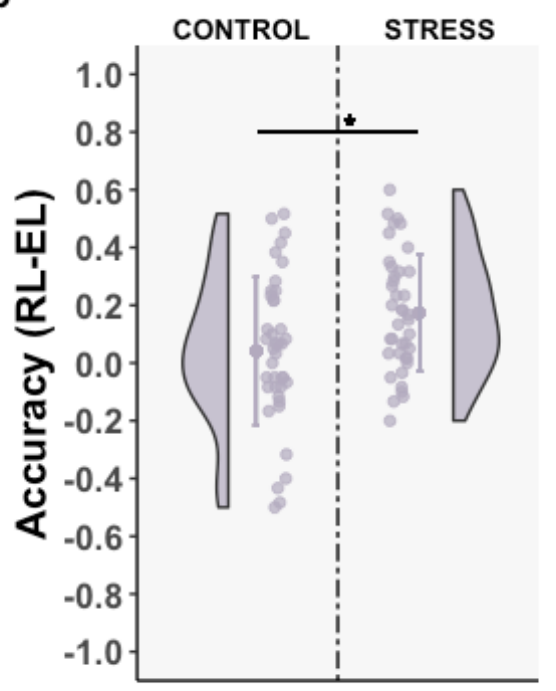

C

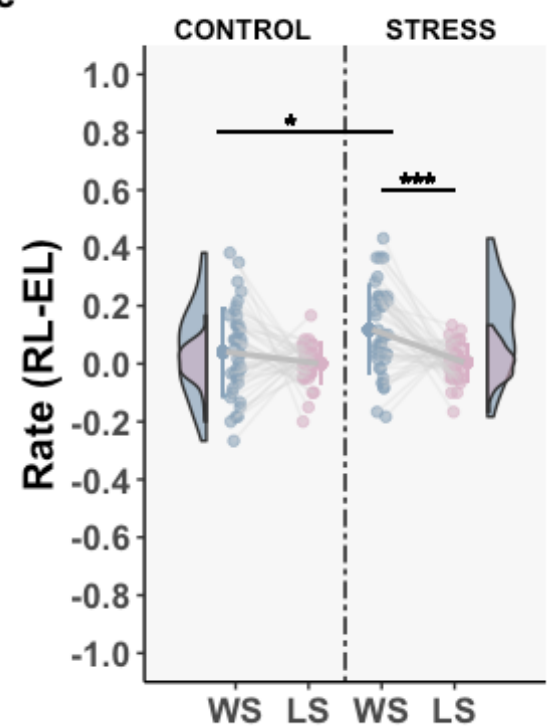

Acute stress leads to improved benefit versus cost learning.

Panel a: Average accuracy (choices of the optimal stimulus) for RL and EL trials, for each

condition separately. Panel b: RL-EL average accuracy difference scores. Panel c:

Win-stay $\left(\mathrm{WS}_{\mathrm{RL}}-\mathrm{WS}_{\mathrm{EL}}\right)$ and lose-shift $\left(\mathrm{LS}_{\mathrm{RL}}-\mathrm{LS}_{\mathrm{EL}}\right)$ difference scores for each condition

displayed. In panel a, the top line indicates a significant Condition-by-Trial type interaction.

Significant differences are denoted by asterisks $(*: p<0.05, * *: p<0.01, * * *: p<0.001)$.

Source files of task performance data used for the analyses are available in the Figure 3 -

Source Data 1.

Asymmetric cost-benefit reinforcement learning biases actions in acute stress subjects

265 During a surprise 64-trial test phase (D. Hernaus, Gold, Waltz, \& Frank, 2018), we asked

266 participants to discriminate original and novel combinations of stimuli on the basis of reward value or action cost without receiving feedback ( $n=16$ trials for original combinations; $n=48$ 
assess learned choice tendencies without having to arbitrarily choose a given number of final surprise test phase trials involving the original four pairs [one-sample t-test against chance; control $_{\mathrm{RL}}: t(39)=8.73, p<0.001$; controlel $_{\mathrm{EL}}: t(39)=3.72, p=0.002$; $\operatorname{stresSRL}_{\mathrm{RL}}: t(39)=13.54$, preference for the optimal stimulus.

Although we observed no Condition-by-Trial type (reward value, action cost discrimination) interaction or main effects of Condition for novel stimulus combinations

$279\left[F(1,78)=1.10, p=0.298, n^{2}{ }_{G}=0.01\right.$; stress vs. controls reward value discrimination: $t(75.9)=$ $0.15, p=0.878$; stress vs. controls action cost discrimination: $t(78)=1.77, p=0.080]$, pairwise comparisons revealed that the acute stress group performed better on reward discrimination compared to action cost discrimination trials $[t(39)=-2.23, p=0.032]$, while no-stress controls performed similarly on both trial types $[t(39)=-0.87, p=0.387]$. These results provide some evidence that a reward maximisation-over-action cost minimisation reinforcement learning policy might bias future actions in novel contexts (Figure Supplement 3).

\section{Computational cost-benefit reinforcement learning model: Model Fitting, Selection,}

\section{Demonstrations, and Simulations}

289 To uncover latent mechanisms by which acute stress affects cost-benefit reinforcement

290 learning, we turned to computational cognitive modelling. Trial-by-trial choices of

291 participants were fit to all candidate models described in the Materials and Methods (see

292 Model Space). To calculate model fit, log likelihood was updated trial-wise by the log of the 293 probability of the observed choice, calculated via a softmax rule (see Materials and Methods, 
equation 6), and best-fitting parameters were identified using fmincon in MATLAB v.2019B

297 http://www.fil.ion.ucl.ac.uk/spm/software/spm12/) using the Akaike Information Criterion

298 (AIC) as a fit statistic that penalizes for the number of model parameters (Myung, Tang, \&

299 Pitt, 2009), suggested that the 2LR_ $\gamma$ model was the most likely model, as indicated by the

300 protected exceedance probability (pxp, $\varphi=0.99)$ (Rigoux, Stephan, Friston, \& Daunizeau,

$3012014)$ and expectation of the posterior [p(r|y), 0.70] (Figure 4a for $\mathrm{p}(\mathrm{r} \mid \mathrm{y})$ of all candidate

302 models). We note that 2LR_ $\gamma$ remained the most likely model when we considered additional

303 models with greater redundancy and/or lesser biological plausibility (e.g., models with all

304 combinations of reward value/action cost discounting and weight parameters).

The 2LR_ $\gamma$ model contains separate learning rates that weight the importance of RPEs

307 parameter $(\beta)$, which in previous work could account for performance on a conceptually

308 similar cost-benefit learning task (Skvortsova et al., 2014). To demonstrate the effect of

309 changes in parameters values on choice preferences within the 2LR_ $\gamma$ architecture, we first

310 simulated choices from 50 artificial agents (averaged across 10 repetitions) performing the

311 reward maximization/action cost minimization reinforcement learning task using a range of

312 parameter values. As expected, greater values of $\alpha_{R}$ and $\alpha_{E}$ primarily impacted the speed of

313 RL and EL choice preferences, while low values of $\gamma$ lead to asymmetric choice preferences

314 through discounting of action cost, and lower values of $\beta$ lead to non-selective increases in

315 random sampling (Figure 4b).

316 In post hoc simulations, i.e., generating participant choices using the obtained

317 parameters, we additionally observed moderate-to-high correlations between simulated and

318 empirical RL/EL for the acute stress and no-stress control group $\left[\rho_{\text {RL_control }}=0.55, p<0.01\right.$; 
$\rho_{\text {RL_stress }}=0.84, p<0.01 ; \rho_{\text {EL_control }}=0.56, p<0.01 ; \rho_{\text {EL_stress }}=0.77, p<0.01$; see Figure

320 Supplement 4], although the canonical performance difference in RL versus EL accuracy was

321 not selective to the acute stress group $\left[t_{\text {control }}(39)=-6.72, p<0.001 ; t_{\text {stress }}(39)=-6.01, p<0.001\right]$.

322 However, after we fixed $\beta$ and $\gamma$ to group-level averages, to better demonstrate the effect of

323 group differences in the learning rate parameters, we recovered a small but significant

324 simulated difference in RL versus EL performance for the acute stress group $[t(39)=2.27$,

$325 p=0.029]$, which was not predicted in the no-stress control group $[t(39)=0.91, p=0.367]$

326 (Condition-by-Trial Type interaction: $\left[F(1,78)=0.77, p=0.38, n^{2}{ }_{G}=0.006\right]$ ) (Figure $4 \mathrm{c}$ for

327 empirical versus simulated data, averaged across 100 repetitions per subject).

328 Importantly, even if a given model is the most likely one based on model fitting and

329 post hoc simulation results from the entire sample, there is still the possibility that different

330 models can better explain task performance in the no-stress control and acute stress

331 condition. When repeating BMS for each condition separately, 2LR_ $\gamma$ was the most likely

332 model in the no-stress control group $[\varphi=0.99, \mathrm{p}(\mathrm{r} \mid \mathrm{y})=0.83]$, while for acute stress subjects

333 2LR_ $\gamma$ was not convincingly the most likely model $[\varphi=0.47 \mathrm{p}(\mathrm{r} \mid \mathrm{y})=0.46]$. Here, the 2LR

334 model (containing $\alpha_{R}, \alpha_{E}$, and $\beta$ parameters) was equally likely to be the optimal model

$335[\varphi=0.53 \mathrm{p}(\mathrm{r} \mid \mathrm{y})=0.47]$. Post-hoc simulations from the 2LR model also correlated with actual

336 data, both for no-stress control $\left[\rho_{\mathrm{RL}}=0.65, p<0.001 ; \rho_{\mathrm{EL}}=0.60, p<0.001\right]$ and acute stress

337 participants $\left[\rho_{\mathrm{RL}}=0.81, p<0.001 ; \rho_{\mathrm{EL}}=0.75, p<0.001\right]$.

338 Similar to the 2LR_ $\gamma$ model (results discussed in next section), the 2LR model

339 seemingly also explained stress-induced changes in cost-benefit reinforcement learning via

340 changes in learning rates; in the 2LR model, the acute stress group exhibited greater values of

$341 \alpha_{\mathrm{R}}$ versus $\alpha_{\mathrm{E}}(\mathrm{t}(39)=2.65, p=0.01)$, while no-stress control subjects did $\operatorname{not}(t(39)=0.69$,

$342 p=0.50)$ (Condition-by-Learning Rate interaction: $\left[F(1,78)=2.88, p=0.094, n^{2}{ }_{G}=0.01\right]$. The

343 difference in learning rates between 2LR_ $\gamma$ (where $\alpha_{R}$ and $\alpha_{E}$ are similar for the acute stress 
344 group, see next section) and 2LR (where $\alpha_{R}>\alpha_{E}$ for the acute stress group) can be explained

345 by the absence of discounting parameter $\gamma: 2 \mathrm{LR}$ is a special case of $2 \mathrm{LR} \_\gamma$, where $\gamma=1$, and

346 thus asymmetric effects of acute stress on reward value maximization and action cost

347 minimization can only be explained by dissimilarity in learning rates.

348 Although the effects of acute stress on reward value and action cost learning rates are 349 opposite in 2LR_ $\gamma$ versus 2LR architectures, these results bolster our confidence in the overall model space, as well as the interpretation that acute stress primarily impacts reward

351 value and action cost learning rates, and not discounting. The observations that I) $2 L_{-} \gamma_{\text {fit }}$

352 better in the entire group of participants, II) 2LR is fully contained within the 2LR_ $\gamma$ model, and III) 2LR_ $\gamma$ displayed good recoverability (see below) motivated our choice to focus on the 2LR_ $\gamma$ model.

In model recoverability analyses i.e., re-fitting the simulated data from the model to

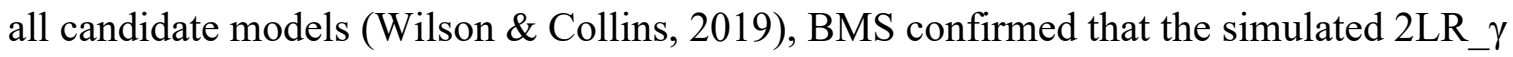
data (that is, simulations without fixed parameters) were most likely to be generated from 2LR_ $\gamma[\varphi=0.99, p(r \mid y)=0.71]$.

To assess the stability of 2LR_ $\gamma$ parameters, we repeated model fitting using a

360 Bayesian hierarchical model fitting approach consisting of two steps, as described previously

361 (Daw, 2011; Frey, Frank, \& McCabe, 2019). In the first step we fit the 2LR_ $\gamma$ model to trial362 wise choices to obtain subject-specific parameters; in a second step we again fit the model to 363 trial-wise choices, but this time we used the group-level average and covariance matrix of 364 every parameter as priors, thereby shrinking the parameter search space. Motivated by recent work showing that group-specific priors, compared to a single prior for the entire sample, can

366 better account for between-group differences in task performance, as well as improve

367 parameter robustness and recoverability (Valton, Wise, \& Robinson, 2020), we used separate 368 mean and covariance matrices for the acute stress and no-stress control groups. 
370 parameter estimates after Bayesian hierarchical model fitting see Figure Supplement 5).

371 Similar to post hoc simulations using parameters from the non-hierarchically fit 2LR_ $\gamma$

372 model, we observed moderate-to-high correlations between empirical and simulated data

373 using parameters obtained from the hierarchically fit model $\left[\rho_{\mathrm{RL} \_ \text {control }}=0.65, p<0.01\right.$;

$374 \rho_{\text {RL_stress }}=0.84, p<0.01 ; \rho_{\text {EL_control }}=0.37, p=0.02 ; \rho_{\text {EL_stress }}=0.78, p<0.01$; see Figure

375 Supplement 6]. All in all, these results confirm parameter stability within the 2LR_ $\gamma$

376 architecture.

377 In light of model fitting results, post-hoc simulations, model and parameter

378 recoverability analyses, we used parameters and trial-by-trial predictions of the non-

379 hierarchically fit 2LR_ $\gamma$ model in all analyses reported below. 
a

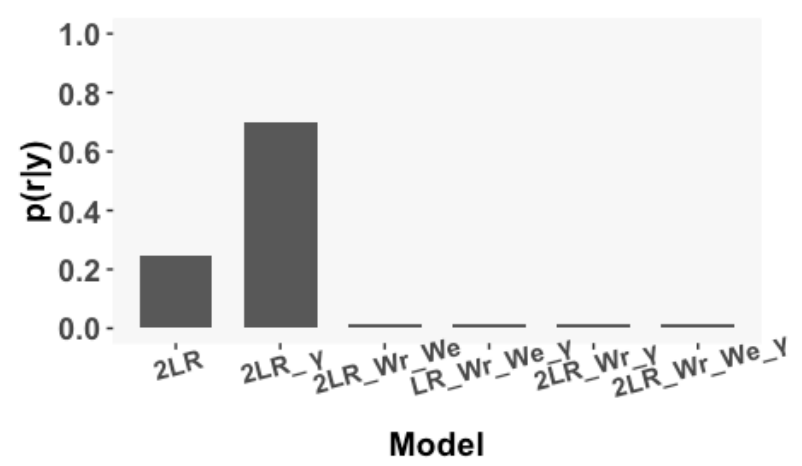

c

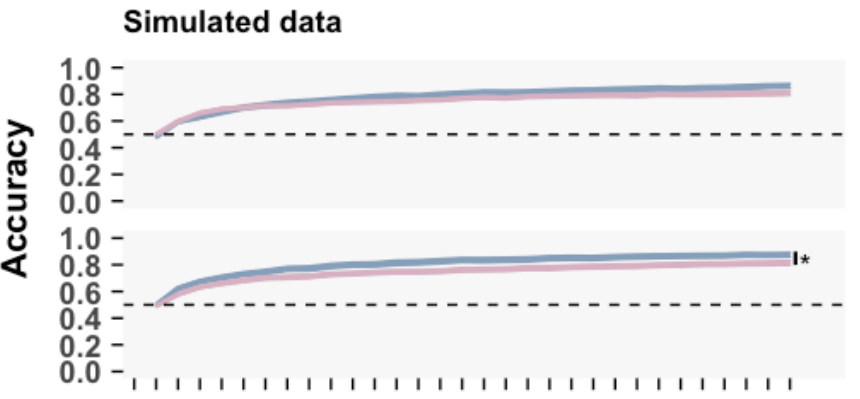

b

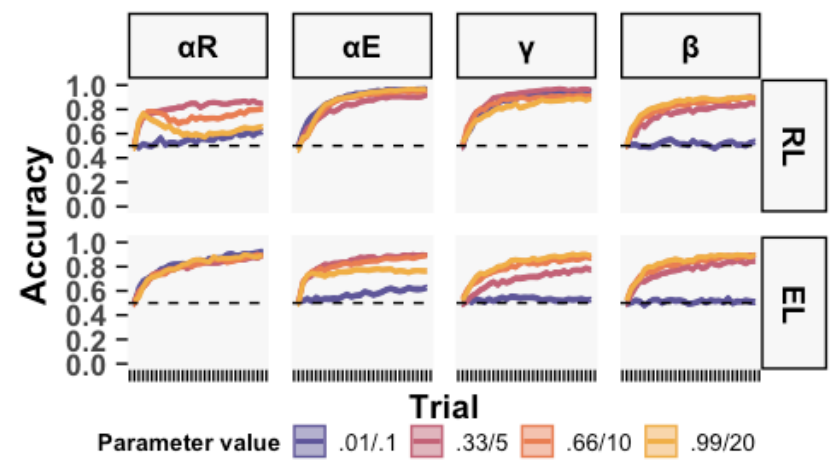

Actual data

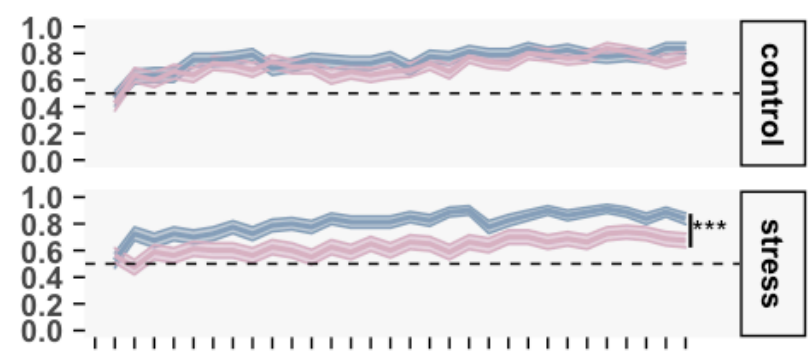

Trial
Trial type $\square \mathrm{RL} \boxminus \mathrm{EL}$

382 Model selection, demonstrations, and post hoc simulations of the winning model.

383 Panel a: Expectation of the posterior for all candidate models. Panel b: Model

384 demonstrations. To demonstrate how different parameter values within the 2LR_ $\gamma$

385 architecture impact choice preferences for the optimal stimulus ("accuracy"), $\alpha_{R}, \alpha_{E}$, and $\gamma$

386 were set to $0.01 / 0.33 / 0.66 / 0.99$, while $\beta$, a non-linear parameter, was set to $0.1 / 5 / 10 / 20$.

387 Parameter effects were always demonstrated for a single parameter (columns), while all other

388 parameter values were kept constant ( $\alpha_{R}$ and $\left.\alpha_{E}=0.25, \gamma=1, \beta=25\right)$. Greater values of $\alpha_{R}$ and

$389 \alpha_{E}$ selectively increase the speed with which the agent develops a preference for the optimal

390 RL and EL stimulus, respectively. Lower values of $\gamma$ produce an asymmetric decision-

391 making policy that emphasises reward value over action cost, leading to better performance

392 on RL versus EL trials, while greater values of $\gamma$ correct this asymmetric choice bias. Finally,

393 greater $\beta$ values lead to more deterministic sampling of optimal stimuli. Panel c: Post-hoc 
simulations after fixing $\beta$ and $\gamma$ to group-level averages. Coloured lines represent mean \pm SD.

395 Dashed lines denote chance level (0.5). *: $p<0.05, * *: p<0.01, * * *: p<0.001$.

396

397 Acute stress selectively reduces the difference between reward and action cost learning

\section{8 rates}

399 Comparing 2LR_ $\gamma$ parameters between conditions, we observed a significant Condition-by-

400 Learning Rate $\left(\alpha_{\mathrm{R}}, \alpha_{\mathrm{E}}\right)$ interaction $\left[F(1,78)=6.42, p=0.01, n^{2}{ }_{G}=0.03 ; 95 \%\right.$ highest density

401 interval (HDI) for Bayesian mixed ANOVA $=-0.405$ to -0.023 , mean $=-0.219]$. with greater

402 EPE relative to RPE learning rates in no-stress control participants $[t(39)=-4.75, p<0.001]$,

403 while learning rates in the acute stress group did not significantly differ $[t(39)=-1.61$,

$404 p=0.116]$. No between-group differences in $\alpha_{\mathrm{R}}$ and $\alpha_{\mathrm{E}}$ or in the other parameters $(\gamma, \beta)$ were

405 observed (all p-values $>0.05)$ (Figure 5).

Paradoxically, symmetric reward value and action cost learning rates in the presence

407 of lower values of $\gamma$ will lead to more efficient RL compared to EL. This is because lower

408 values of $\gamma$ bias decisions towards reward value (via greater discounting of action cost) and

409 similar absolute values of $\alpha_{\mathrm{R}} / \alpha_{\mathrm{E}}$ will not counteract this bias. Asymmetric learning rates

$410\left(\alpha_{E}>\alpha_{R}\right)$ in combination with lower values of $\gamma$, however, will lead to more symmetric

411 performance on RL and EL trials via more efficient updating of action cost versus reward

412 expectations. This interpretation is supported by our demonstration of model parameters

413 (Figure $4 \mathrm{~b}$ ) and post hoc simulations (Figure 4c), as well as the observation that lower values

414 of $\gamma$ (i.e., greater action cost discounting) were associated with greater learning rate

415 asymmetry $\left(\alpha_{\mathrm{E}}>\alpha_{\mathrm{R}}\right.$; more efficient EL) in no-stress controls $(\rho=-0.40, p=0.040)$, who

416 displayed similar RL and EL performance. These results demonstrate that, in a context where

417 all decisions involve a potential cost and benefit, acute stress selectively reduces the

418 difference between EPE and RPE learning rates, while leaving action cost discounting and 
419 choice stochasticity unaffected. The direction of the change in learning rates (i.e., greater

420 similarity) implies a stress-induced failure to modulate learning rates in the service of

421 overcoming an asymmetric choice bias that emphasises reward value.

422 In analyses using posterior parameters obtained from the hierarchically fit model, we

423 recovered the key Condition-by-Learning Rate interaction (95\% HDI for Bayesian mixed

424 ANOVA $=-0.406$ to -0.128 , mean $=-0.269$ ) [and acute stress and no-stress control subjects

425 differed from each other on $\alpha_{\mathrm{E}}(95 \% \mathrm{HDI}=0.0841$ to 0.281 , mean $=0.183)$ but not $\alpha_{\mathrm{R}}(95 \%$

$426 \mathrm{HDI}=-0.186$ to 0.0102 , mean $=-0.0872)]$ (Figure Supplement 7 ). Similar to the non-

427 hierarchically fit parameters, acute stress and control subjects did not differ on posterior

428 estimates of $\gamma(95 \% \mathrm{HDI}=-0.0914$ to 0.139 , mean $=0.0182)$ and $\beta(95 \% \mathrm{HDI}=-0.0331$ to

4290.213 , mean=0.0895).

430 


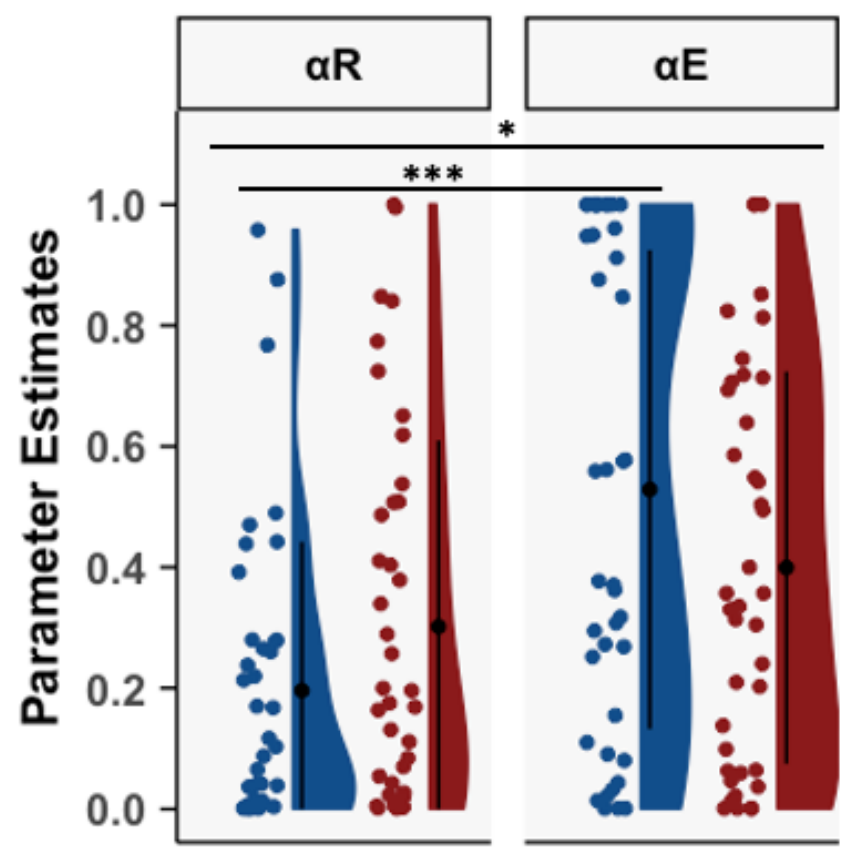

433

434

435 controlled by ascending midbrain modulatory systems that play a role in value-based

444 decision-making and the acute stress response (Arnsten, 2015; Hermans et al., 2011; Joshi,

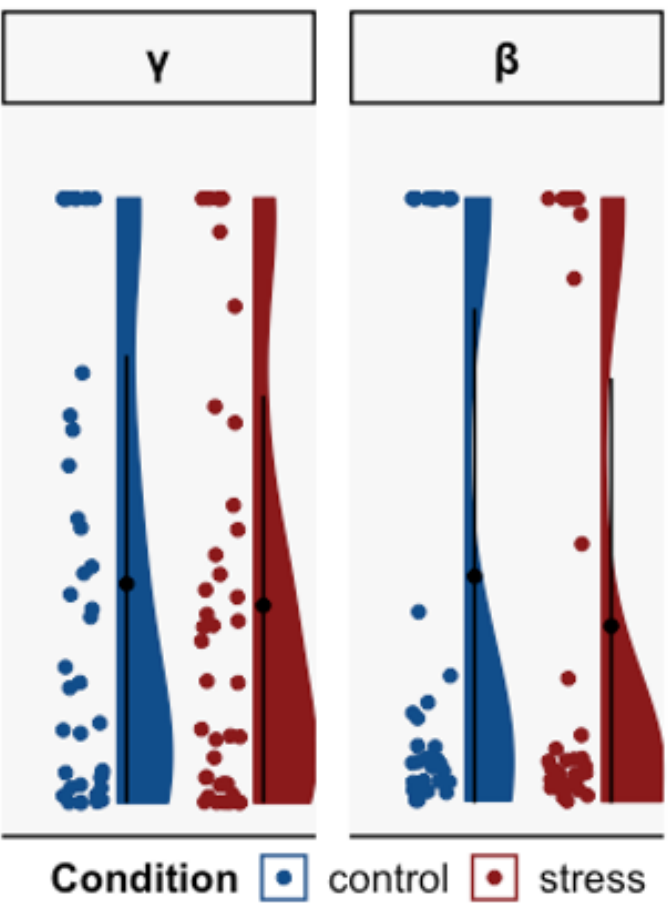

Acute stress reduces the difference between reward and effort prediction error learning rates.

Free parameters $(\alpha \mathrm{R}, \alpha \mathrm{E}, \gamma, \beta)$ of the winning $2 \mathrm{LR} \_\gamma$ model for both groups. Black lines denote means $\pm \mathrm{SD}$, dots represent individual data points, and the violin-like shape denotes distribution and frequency of the data. $*: p<0.05, * *: p<0.01, * * *: p<0.001$.

\section{Pupil size fluctuations track asymmetric cost-benefit reinforcement learning during} acute stress

We employed pupillometry to better understand whether task-relevant computational processes may be encoded by fluctuations in pupil dilation, which are thought to be Li, Kalwani, \& Gold, 2016)

Li, Kalwani, \& Gold, 2016). 
In model-free analyses - that is, comparing bins of pupillometry data between

447 conditions - we observed no main effect of Condition on pupil size fluctuations during

448 choice, effort outcome, and reward outcome epochs, suggesting that acute stress was not

449 associated with more general changes in pupil size (all bin-level $p>0.05$; Figure 6 , a. Model-

450 free; Figure Supplement 8 for all effort trials).

Next, we conducted model-based pupillometry analyses (Lawson, Bisby, Nord,

452 Burgess, \& Rees, 2020) to understand how trial-wise estimates of computational processes of

453 interest were encoded by fluctuations in pupil size. These analyses revealed effects of

454 Condition on pupil encoding of subjective decision value, EPEs and RPEs (Figure 6, b.

455 Model-based) in a manner commensurate with task performance results. First, immediately

456 prior to the stimulus choice, acute stress reduced pupil encoding of subjective decision value,

457 as evidenced by the absence of an association between pupil size and subjective decision

458 value (control $>$ stress; stress n.s., control $>0$ ). Second, briefly after the presentation of effort

459 avoidance outcomes, both groups exhibited different pupil size-EPE associations, with no-

460 stress controls showing a non-significant numerically positive association between pupil size

461 and action cost prediction errors (control $>$ stress, both groups n.s. different from 0 ). In model-

462 based analyses using all effort outcome trials, we were not able to uncover group differences

463 in pupil encoding of EPEs, which were likely eclipsed by prominent grip force-related effects

464 on pupil size (Figure Supplement 8). Third, during the reward outcome phase, acute stress

465 participants exhibited greater positive associations between pupil size and RPEs compared to

466 no-stress controls (stress $>$ control, stress $>0$, control n.s.). The average pupil size-RPE slope

467 for bins in which no-stress control and acute stress participants differed (Figure 6b) correlated

468 significantly with stress-induced changes in $\operatorname{SBP}\left[\rho_{\text {stress }}(38)=-0.41, p(\right.$ permutation $\left.)=0.019\right]$

469 and PANAS negative affect changes $\left[\rho_{\text {stress }}=-0.46, p\right.$ (permutation $\left.)=0.005\right]$ in the stress group. 
471 RPEs imply that the ascending neuromodulatory systems may have facilitated a stress-

472 induced shift in asymmetric cost versus benefit learning.

473

$474 \quad$ Figure 6

a. Model-Free

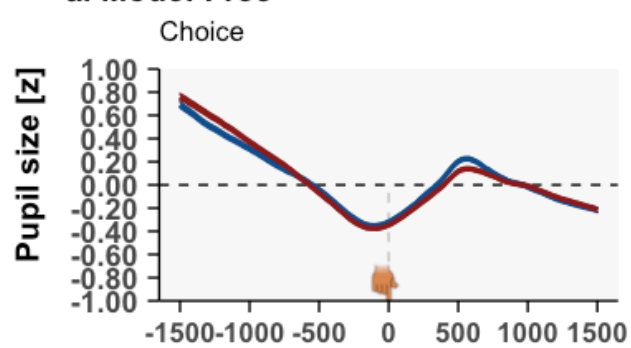

\section{b. Model-Based}

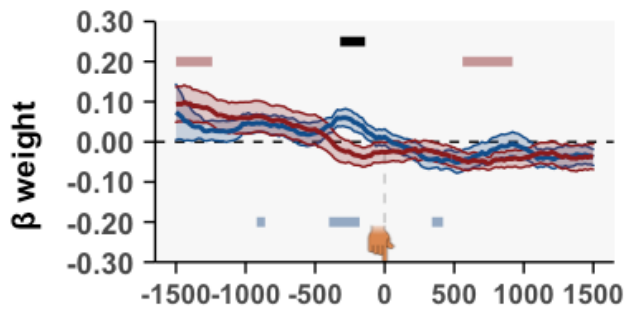

475

476

477

478

479

480

481

482

483

484

485

value during acute stress .
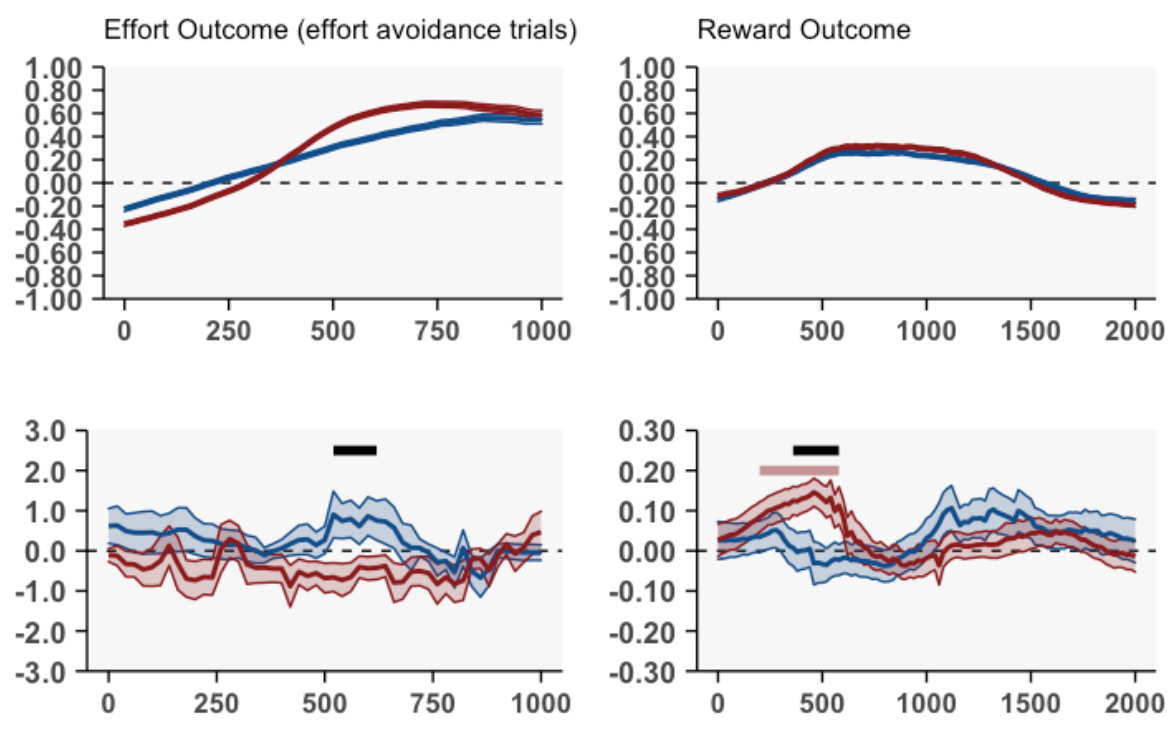

Time [ms]

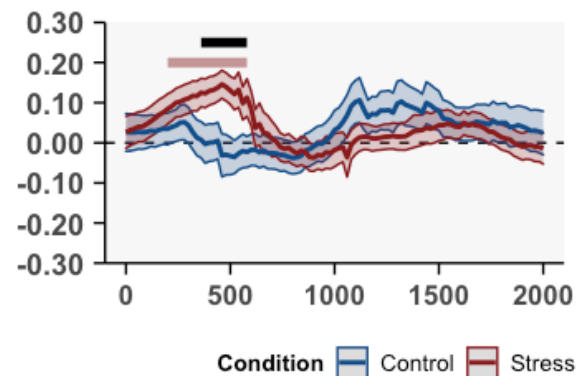

\section{Model-based analysis reveals altered pupil encoding of prediction errors and decision}

a. Model-free analyses of pupil size during choice, effort outcome, and reward outcome phase revealed no main effect of Condition (no-stress control, acute stress). b. Model-based analyses revealed a stress-induced shift in pupil encoding of subjective decision value (left), action cost prediction errors (middle) and reward prediction errors (right). Black line indicates significant main effect of Condition; blue and red line indicate significance against zero for no-stress control and acute stress groups, respectively (cluster and bin level $\alpha_{\text {permute }}<0.05,2000$ permutations). Group differences in pupil encoding of action cost and reward prediction errors were observed at similar times (note the $\mathrm{x}$-axis differences for effort 
outcome and reward outcome epochs). Source files of pupillometry data used for the analyses

487 are available in Figure 6 - Source Data 2.

488

\section{Discussion}

490 Stress-induced alterations in adaptive decision-making are commonly studied using

491 paradigms that isolate positive and negative reinforcement, such as the receipt of a reward or

avoidance of a loss. However, it remains poorly understood how acute stress affects the

493

complex process that entails learning about costs and benefits, a critical and pervasive feature

494 of everyday decisions. Participants completed a paradigm in which all actions (stimulus

495 choices) contained a potential cost (exerting physical effort) and a financial benefit $(€ 0.20)$.

496 Crucially, acute stress induced a shift in reinforcement learning strategies that improved

497 maximization of monetary rewards relative to minimisation of energy expenditure. When

498 presented with novel stimulus arrangements and in the absence of feedback, individuals in

499 the acute stress condition, moreover, exhibited better discrimination of stimulus reward value

500 compared to action cost.

$501 \quad$ Relative improvements in reward versus action cost learning align well with previous

503 Lighthall et al., 2013; Petzold et al., 2010), although such effects may depend on stressor

504 timing (Joëls, Pu, Wiegert, Oitzl, \& Krugers, 2006), stressor type (Carvalheiro et al., 2020),

505 and/or sample characteristics (Evans \& Hampson, 2015; Morris \& Rottenberg, 2015). While

506 reports on action cost learning during acute stress are scarce, acute exposure to stress in

507 rodents impairs cost-benefit decisions via a selective change in sensitivity to physical effort, a

508 process mediated by corticotropin-releasing factor and dopamine (Bryce \& Floresco, 2016).

509 Our analyses of win-stay/lose-shift rates indicate that asymmetric cost-benefit learning can be 
510 driven by a relative increase in the sensitivity to monetary gains compared to the avoidance

511 of costly deterrents.

512 How might maximization of reward value take precedence over minimisation of

513 action cost? Acute stress leads to a redistribution of finite cognitive resources (Hermans et

514 al., 2014): this process limits the availability of computationally intensive strategies,

515 including working memory (Otto, Raio, Chiang, Phelps, \& Daw, 2013; Qin, Hermans, van

516 Marle, Luo, \& Fernández, 2009) and goal-directed instrumental actions (Lars Schwabe \&

517 Wolf, 2011). Assuming that acute stress does not merely increase random responding - which

518 we verified via the choice stochasticity model parameter - a computationally cheap heuristic

519 in our task should present itself as better learning for one modality over the other. Increased

520 energy availability (Hermans et al., 2014), insensitivity to aversive stimuli (Timmers et al.,

521 2018), and impaired aversive value updating (Raio et al., 2017) under stress may have

522 reduced the ability - or urgency - to dedicate cognitive resources to strategies that minimize

523 action cost. Importantly, effort expenditure increases the perceived value of rewards

524 (Hernandez Lallement et al., 2014; Inzlicht, Shenhav, \& Olivola, 2018). Thus, frequent

525 expenditure of physical effort, due to suboptimal action cost learning, may increase the

526 perceived value of rewards, and thus tilt learning towards the maximization of reward value.

Using a computational model of reinforcement learning (Skvortsova et al., 2017;

528 Skvortsova et al., 2014), we confirmed that biased cost-benefit learning can arise when

529 inappropriate (i.e. more similar) importance is afforded to teaching signals that convey

530 information about reward value (RPEs) and action cost (EPEs). Humans display presumably

531 instinctive biases, such as more efficient learning from better-than-expected outcomes

532 (Lefebvre, Lebreton, Meyniel, Bourgeois-Gironde, \& Palminteri, 2017) (compared to worse-

533 than-expected outcomes) and asymmetric "Go"/approach learning (Guitart-Masip et al.,

534 2012) (compared to "No-Go"/avoidance learning), the latter being a bias that is also 
modulated by acute stress (de Berker et al., 2016). From this perspective, no-stress controls,

biased towards the reward value of actions (captured by action cost discounting parameter $\gamma$ ).

539 Paradoxically, when decisions are by default tilted towards reward value, similar reward and

540 action cost learning rates will facilitate reward learning but hamper action cost learning.

541 Reduced learning rate asymmetry in the presence of action cost discounting may therefore

542 represent a computational reformulation of a heuristic that is employed when cognitively

543 demanding learning strategies are unavailable and the policy towards energy expenditure is

544 more liberal, such as during acute stress.

Importantly, stress-induced changes in task performance may crucially depend on the release of catecholamines in neural circuits that support motivation and learning. Dopamine's actions at D1 and D2 receptors in the basal ganglia mediate approach and avoidance learning (Frank, Seeberger, \& Reilly, 2004), and acute stress can improve associative learning by augmenting reward-evoked DA bursts in selective striatal subdivisions (Stelly, Tritley,

550 Rafati, \& Wanat, 2020). Dopamine's enhancement via L-DOPA administration, moreover, improves reward but not action cost learning (Skvortsova et al., 2017). To the degree that pupillometry can be considered a proxy measure of activity of ascending neuromodulatory systems, these findings are consilient with greater encoding of RPEs by pupil size

554 fluctuations during acute stress. Negative correlations between SBP and PANAS negative

555 affect and RPE-pupil size slopes suggest that primarily moderately stressed participants displayed a preference for maximizing reward value, which might be consistent with an inverted U-shape relationship between cognitive performance and DA transmission which is modulated by stress (Arnsten \& Goldman-Rakic, 1998; Baik, 2020). Noradrenaline, however, mobilizes available energy to complete effortful actions and locus coeruleus neurons track 
energy expenditure (Varazzani et al., 2015). Stress-induced sAA concentrations, increased heart rate, and group differences in the association between pupil size fluctuations and EPEs

562 all point to the involvement of the noradrenaline system. Thus, our model-based pupillometry

563 and stress-induction results hint at stress-sensitive dopaminergic and noradrenergic

564 mechanisms that may regulate cost and benefit learning, which could be explored in future work using targeted pharmacological approaches.

567 psychopathology. While asymmetric cost-benefit learning during acute stress may be beneficial to reach a desired goal state (e.g., safety) despite high action cost, such strategies could also be maladaptive. For example, stress exposure can lead to drug or smoking relapse (L. Schwabe, Dickinson, \& Wolf, 2011), a context in which reward value and action cost may be misaligned. Cost-benefit reinforcement learning may provide a useful framework to test hypotheses regarding stress-related impairments in learning and decision-making. effort expenditure greatly reduced our power to detect robust associations between EPE encoding and pupil size fluctuations. Future studies should, therefore, consider a temporal delay between effort outcome and effort expenditure phases. Second, while our computational model was able to recover overall task performance patterns in both groups,

578 such effects were subtle and dependent on the contribution of other (non-learning)

579 parameters, which may highlight the importance of interindividual differences in model 580 parameters.

To summarize, we present evidence of asymmetric effects of acute stress on cost

582 versus benefit reinforcement learning during acute stress, which computational analyses of 583 task behaviour explain as a failure to assign appropriate importance to RPEs versus EPEs, 584 and our model-based pupillometry tentatively link to activity of ascending midbrain 
neuromodulatory systems. These results highlight for the first time how learning under acute

587 things.

Materials and Methods

590

Participants

591 Adult participants were recruited via paper and online advertisements. All participants were screened for a DSM-5 psychiatric and/or neurological disorder, substance use, endocrine and/or vascular disorder, abnormal BMI $(>40$ or $<18)$, smoking and drinking $(>10$ cigarettes/units per week), psychotropic medication use (lifetime) and hormonal contraceptive use (current; female participants only). All participants completed the $\sim 2$-hour experiment between 12:00h and 18:00h to minimize diurnal cortisol fluctuations (Bailey \& Heitkemper, 2001). Participants were instructed to refrain from alcohol (starting the evening before the day of the experiment), smoking, food, caffeine intake, strenuous physical activity and brushing their teeth (all $>2 \mathrm{hr}$ prior to experiment), which was verified verbally at the start of the session. Four participants were excluded due to an equipment failure $(n=4)$. Three participants quit during stress-induction $(n=2)$ or task procedures $(n=1)$. Because chancelevel performance on reinforcement learning tasks might indicate a successful manipulation, a lack of motivation, or a failure to comprehend the task instructions, participants that

604 performed at or below chance level (0.5) on both RL and/or both EL pairs near the end of the experiment (final 10 presentations) were excluded ( $\mathrm{n}=13 ; 6$ acute stress, 7 no-stress control).

606 Including these participants did not alter our key finding that acute stress was associated with 607 asymmetric cost versus benefit learning (see Results). Pupillometry and neuroendocrine data 608 were not processed further for these participants. The study was approved by the ethics 609 committee of the Faculty of Psychology and Neuroscience, Maastricht University (ERCPN- 
610 197_03_08_2018) and carried out in accordance with the Declaration of Helsinki.

611 Participants were remunerated in gift vouchers or research participation credits. Task

612 earnings were paid out in gift vouchers.

613

\section{Acute stress induction}

615 The MAST is a validated stress-induction paradigm combining both psychological and

616 physiological stressors, and robustly increases neuroendocrine, physiological, and subjective

617 indices of acute stress (Smeets et al., 2012). During a 5-min preparation phase, participants

618 are instructed about the upcoming task via oral and visually displayed instructions, followed

619 by a 10 -min stress-induction phase consisting of alternating blocks of cold-water immersion

620 (non-dominant hand; $2^{\circ} \mathrm{C}$ ) and backward counting in steps of 17 (while receiving negative

621 evaluative feedback from an experimenter), with a (non-recording) camera continuously

622 directed at the participant's face, which was displayed to the participant on a second display.

623 During the MAST no-stress control condition, participants immerse their hand in lukewarm

624 water $\left(36^{\circ} \mathrm{C}\right)$ and perform simple mental arithmetic, e.g., counting from 1 to 25 , without

625 receiving feedback or fake camera recordings.

626

627 Neuroendocrine, physiological and subjective stress measurements

628 sCORT and sAA were collected to measure stress-induced increases in hypothalamic-

629 pituitary-adrenal (HPA) axis and sympathetic-adrenal-medullary (SAM) axis activity,

630 respectively (Dickerson \& Kemeny, 2004; Koh, Ng, \& Naing, 2014). Saliva samples were

631 obtained using synthetic Salivette ${ }^{\circledR}$ devices (Sarstedt, Etten-Leur, the Netherlands) during 3-

632 min sampling periods at 6 time points. A baseline sample was collected 10 min prior to the

633 MAST (baseline: $\left.\mathrm{t}_{1}=\mathrm{t}_{-10}\right)$ and five samples post-MAST $\left(\mathrm{t}_{2}=\mathrm{t}_{+00}, \mathrm{t}_{3}=\mathrm{t}_{+10}, \mathrm{t}_{4}=\mathrm{t}_{+20}, \mathrm{t}_{5}=\mathrm{t}_{+30}, \mathrm{t}_{6}=\right.$

$\left.634 \mathrm{t}_{+40}\right)$. SAA assessments were obtained only for $\mathrm{t}_{1}-\mathrm{t}_{4}$, due to the rapid decay of sAA post-stress 
induction (Dennis Hernaus, Quaedflieg, Offermann, Casales Santa, \& van Amelsvoort, 2018;

Nater et al., 2005). For all participants, $t_{2}$ marked the starting point for the reward value completion of each session. SCORT and sAA levels were determined using a commercially available luminescence immune assay kit (IBL, Hamburg, Germany) and kinetic reaction assay (Salimetrics, Penn State, PA, USA), respectively. assessed at $\mathrm{t}_{1}$ and $\mathrm{t}_{2}$ using an OMRON M4-I blood pressure monitor (OMRON Healthcare

644 Europe B.V., Hoofddorp, The Netherlands). Subjective affect ratings were assessed at $\mathrm{t}_{1}$ and $\mathrm{t}_{2}$ using the 20-item Positive and Negative Affect Scale (PANAS) (Watson, Clark, \& 646 Tellegen, 1988).

\section{Reward maximization versus action cost minimization reinforcement learning task}

649 All participants completed a probabilistic stimulus selection paradigm during which they 650 learned to select stimuli with high reward value (20 Eurocents) and avoid stimuli with high action cost (exerting force above a pre-calibrated individual threshold for a duration of $3000 \mathrm{~ms}$ ). This reinforcement learning task is conceptually similar to a previously-validated probabilistic action selection task that has been employed to study the neural signatures of reward and effort prediction errors and dopaminergic drug effects on reward-effort computations (Skvortsova et al., 2017; Skvortsova et al., 2014). The paradigm was designed in PsychoPy v3.0.0b11 (Peirce et al., 2019) and presented on a 24" monitor (iiyama ProLite b2483HSU). Physical effort (in $\mathrm{mV} / \mathrm{kgf}$ ) was registered using a hand-held dynamometer in combination with a transducer amplifier (DA100C) and data acquisition system (MP160; all manufactured by BIOPAC Systems, Inc). Individual effort thresholds used throughout the 
task were obtained by calculating 50\% of each participant's maximal voluntary contraction

661

(MVC) (Le Heron et al., 2018) reached over three calibration trials by squeezing the

662

663

664

665

666

667

668

669

670

671

672

673

674

675

676

677

678

679

680

681

682

683 dynamometer with the dominant hand.

On each of 120 trials, participants chose between two paired distinct black-and-white images ("stimuli") that were probabilistically associated with both the receipt of a monetary reward and exertion of physical effort (see Figure 1 for a graphical overview). At trial onset, a fixation cross flanked by two images was presented; participants chose one image by pressing the $\mathrm{V} / \mathrm{B}$ button for the left/right option, respectively. A $440 \mathrm{~Hz} / 600 \mathrm{~Hz}$ tone for left/right choice (200ms) was presented to confirm the participant's choice. Next, a thermometer with the command "SQUEEZE" or "DON'T SQUEEZE" was displayed. If participants were required to exert effort, they were instructed to squeeze the dynamometer until the mercury level reached the top. The mercury bar only moved if participants exerted above-threshold levels of force and stopped moving if exerted force fell below. The cumulative above-threshold time was $3000 \mathrm{~ms}$. If no effort production was required, an animation of a rising mercury bar was displayed $(3000 \mathrm{~ms})$. Finally, a screen was presented showing either a $€ 0.20$ coin or a crossed-out coin, indicating no reward (3000ms). Participants learned to choose the optimal (most reward or most effort avoiding) stimulus for four distinct image pairs, 30 presentations each, with yoked reward and action cost contingencies. For $2 / 4$ pairs, participants could regularly acquire rewards by selecting one (optimal) stimulus over the (suboptimal) other (henceforth, "reward learning"/RL pairs), while the probability of having to exert effort was identical for both stimuli. For the other two pairs, choices of one stimulus were more frequently followed by the avoidance of effort ("effort learning"/EL pairs), while the probability of reward was kept constant between both. For all pairs, the probability of the stimulus property that was kept constant (reward/effort) 
was set to a $33.3 \%$ chance of positive outcome upon selection (reward/ effort avoidance) and $66.6 \%$ chance of negative outcome (no reward/effort).

To assess whether any acute stress effects on reward maximization (measured using

687 RL pairs) and effort cost minimization (measured using EL pairs) learning were potentially

688 mediated by task difficulty, we employed different difficulty levels for each RL and EL pair.

689 That is, for one RL and one EL ("easy") pair, a choice for the optimal stimulus was followed

690 by a positive outcome in $83 \%$ (vs. $17 \%$ negative outcome) of all trials ( $83 \%$ negative $/ 17 \%$

691 positive outcome for suboptimal stimulus); for the other RL and EL ("hard") pair a choice for

692 the optimal stimulus was followed by a positive outcome in $70 \%$ (vs. $30 \%$ negative outcome)

693 of all trials (and 70\% negative/30\% positive outcome) for the suboptimal stimulus. This

694 approach allowed us to disentangle whether acute stress primarily impacted domain-specific

695 (RL vs. EL) or general (easy vs. hard) reinforcement learning (the latter which also might

696 involve other cognitive skills that might be beneficial to performance and sensitive to change

697 under stress, such as working memory (Schoofs, Wolf, \& Smeets, 2009). The task

698 contingencies described above were based on extensive pilot tests to identify a reinforcement

699 schedule that would enable us to detect stress-induced improvements and decreases in task

700 performance. We selected task contingencies based on pilot sessions involving a no-stress

701 control condition and chose a reinforcement schedule associated with non-ceiling/floor

702 performance on RL and EL trials.

Following the learning phase, participants completed a surprise test phase, similar to

704 previous work (D. Hernaus et al., 2019; D. Hernaus et al., 2018). This phase consisted of 64

705 trials in which participants were presented with the original four, as well as six novel,

706 stimulus combinations. Participants were asked to choose the stimulus with the highest

707 reward value or the lowest action cost - depending on a coin or thermometer image presented

708 in the middle of the screen - and received no choice feedback. This allowed us to assess 
acquired choice tendencies, as well as generalizability of this information to novel situations.

710 The four original pairs were presented four times (total $n=16$ ) during which we only asked

711 participants to discriminate on the basis on the reward value (for RL) or action cost (for EL).

712 For novel stimulus combinations, we only presented stimuli that differed in reward

713 value/action cost if reward value discrimination/action cost discrimination was assessed (total $714 n=48: n=4$ presentations for the 6 combinations).

715 For every participant, stimuli were randomly assigned to pairs, optimal/suboptimal

716 stimulus orientation was balanced (50\% of all optimal stimulus presentations occurred on the

717 left-hand side) and misleading outcomes (e.g., negative outcomes for optimal stimuli) were

718 equally spaced out across the thirty presentations (and balanced for left/right side). Trial

719 presentation order was pseudo-randomized such that I) a given pair would never be presented

720 more than twice in a row and II) the gap between two presentations of a given pair was never

721 greater than four trials.

722

Prior to performing the actual task and prior before acute stress/no-stress control

723 procedures, participants received standard verbal instructions and completed a 16-trial

724 practice round of the learning phase. Participants were not informed about stimulus-outcome

contingencies; they were only advised to accrue as much money as possible and avoid

exerting unnecessary effort. A $60 \%$ accuracy performance threshold was used to confirm that

727 participants understood the general task procedure. The practice round was repeated if

728 participants failed to reach $60 \%$ accuracy. To prevent learning, we used deterministic

729 stimulus-outcome probabilities and different stimuli.

730

731 Computational cost-benefit reinforcement learning model: model space

732 In an attempt to uncover latent mechanisms by which acute stress affects reward

733 maximization and/or action cost minimization, we turned to cognitive computational 
modelling. We employed a modified reinforcement learning framework based on Rescorla space. and effort outcome for the chosen stimulus at trial $t$. $\mathrm{RPE}_{(\mathrm{t})}$ and $\mathrm{EPE}_{(\mathrm{t})}$, thus, represent the RPE and EPE at trial t, respectively.

In order to allow for the possibility that humans do not calculate the prediction error against the actual outcome but, rather, what the outcome "feels" like (Huys et al., 2013), we considered a scenario in which reward and effort outcomes are first multiplied by a free parameter that captures the weight that reward and effort outcomes receive (" $\mathrm{W}_{\mathrm{R}}$ " and $\mathrm{W}_{\mathrm{E}}$ " in equation $2 \mathrm{~A}$ and $2 \mathrm{~B})$. As the value of these parameters approaches 1, rewards are increasingly valued more positively, and effort more negatively. These parameters, therefore, control the maximum size of the prediction error. 
which prediction errors update choice preferences is represented by $\alpha$, the learning rate determined by outcomes further into the past.

$$
\begin{aligned}
& \mathrm{Q}_{\mathrm{R}(\mathrm{t})}(\mathrm{s}, \mathrm{a})=\mathrm{Q}_{\mathrm{R}(\mathrm{t}-1)}(\mathrm{s}, \mathrm{a})+\alpha_{\mathrm{R}} * \mathrm{RPE}_{(\mathrm{t}-1)}(\mathrm{s}, \mathrm{a})(3 \mathrm{~A}) \\
& \mathrm{Q}_{\mathrm{E}(\mathrm{t})}(\mathrm{s}, \mathrm{a})=\mathrm{Q}_{\mathrm{E}(\mathrm{t}-1)}(\mathrm{s}, \mathrm{a})+\alpha_{\mathrm{E}} * \mathrm{RPE}_{(\mathrm{t}-1)}(\mathrm{s}, \mathrm{a})(3 \mathrm{~B})
\end{aligned}
$$

773 Extensive evidence suggests that organisms use different learning systems for different types

774 of information, including reward value and action cost (Palminteri \& Pessiglione, 2017;

775 Skvortsova et al., 2017; Skvortsova et al., 2014) (equation 3A/B). Thus, the use of separate

776 learning rates for RPEs and EPEs allows for asymmetrical learning about these types of 777 information.

778 While the learning rate controls the speed at which choice preferences are updated,

779 learning rate (nor reward/effort weight) alone does not explain how learned estimates of

780 reward value and action cost may compete at the decision stage (i.e., when participants

781 choose between two stimuli). Agents weight costs against benefits to calculate a subjective

782 decision value (Pessiglione et al., 2017; Skvortsova et al., 2017), which is used to guide 783 choices (equation 4). 


$$
\mathrm{Q}_{(\mathrm{t})}(\mathrm{s}, \mathrm{a})=\mathrm{Q}_{\mathrm{R}(\mathrm{t})}(\mathrm{s}, \mathrm{a})-\mathrm{Q}_{\mathrm{E}(\mathrm{t})}(\mathrm{s}, \mathrm{a})(4)
$$

787 In its simplest form, Q, the subjective decision value of a stimulus is represented by the

788 difference between the expected reward and action cost value at trial $t$ (equation 4)

789 (Skvortsova et al., 2014). However, this particular operationalization of subjective value does

790 not take into account the observation that humans tend to discount or prioritize certain types

791 of information in their decisions (Apps, Grima, Manohar, \& Husain, 2015; Inzlicht et al.,

792 2018). We, therefore, allowed for variation in the calculation of subjective decision value via

793 action cost discounting (equation 5). While discounting rates can be linear or hyperbolic

794 (Hartmann, Hager, Tobler, \& Kaiser, 2013), here we only considered linear discounting in

795 light of previous work using a similar task design (Skvortsova et al., 2017; Skvortsova et al.,

796 2014). As the value of $\gamma$ approaches zero, action cost discounted increases leading the agent

797 to ignore action cost/only utilize reward value to make a decision.

$$
\mathrm{Q}_{(\mathrm{t})}(\mathrm{s}, \mathrm{a})=\mathrm{Q}_{\mathrm{R}(\mathrm{t})}(\mathrm{s}, \mathrm{a})-\gamma^{*} \mathrm{Q}_{\mathrm{E}(\mathrm{t})}(\mathrm{s}, \mathrm{a})(5)
$$

800

801 Once the subjective decision value has been computed, the degree to which

802 participants deterministically sample the optimal stimulus is captured by a softmax decision 803 function (equation 6).

$$
\operatorname{pr}(\mathrm{s}, \mathrm{a})=\exp \left(\mathrm{Q}_{(\mathrm{t})}(\mathrm{s}, \mathrm{a})\right) / \operatorname{sum}\left(\exp \left(\beta^{*} \mathrm{Q}_{(\mathrm{t})}(\mathrm{s})\right)\right)(6)
$$


809 Frank, 2016), $\mathrm{Q}_{(\mathrm{t})}(\mathrm{s}, \mathrm{a})$ is the net value of the chosen option and $\mathrm{Q}_{(\mathrm{t})}(\mathrm{s})$ represents the net

810 values of both stimuli in the pair.

811 Within the above-described model space our predictions of acute stress effects on reward

812 maximization and action cost minimization could, thus, be explained by changes in

813 sensitivity to reward value and/or action cost $\left(\mathrm{W}_{\mathrm{R}}, \mathrm{W}_{\mathrm{E}}\right)$, changes in how much weight $\mathrm{RPEs}$

814 and EPEs are afforded (i.e., learning rates, $\alpha_{\mathrm{R}}, \alpha_{\mathrm{E}}$ ), and/or changes in the discounting of

815 reward value by action cost $(\gamma)$. If acute stress leads to more random responses, such effects

816 should be captured by $\beta$.

817 Based on our predictions and the obtained pattern of results (most notably asymmetrical

$818 \mathrm{RL} / \mathrm{EL}$ performance in the acute stress condition), we considered six candidate models that

819 could capture these various scenarios: I) a model with 2 distinct learning rates for reward and

820 effort $(\alpha \mathrm{R}, \alpha \mathrm{E})[2 \mathrm{LR}]$; II) a model with 2 learning rates $\left(\alpha_{\mathrm{R}}, \alpha_{\mathrm{E}}\right)$ and a discounting parameter

$821(\gamma)\left(2 L_{-} \_\right)$; III) a model with 2 learning rates $\left(\alpha_{R}, \alpha_{E}\right)$, a reward weight $\left(W_{R}\right)$ and an effort

822 weight parameter $\left.\left(\mathrm{W}_{\mathrm{E}}\right)\left(2 \mathrm{LR} \mathrm{W}_{\mathrm{R}_{-}} \mathrm{W}_{\mathrm{E}}\right), \mathrm{IV}\right)$ a model with a single learning rate $(\alpha)$, reward

823 weight $\left(\mathrm{W}_{\mathrm{R}}\right)$, effort weight $\left(\mathrm{W}_{\mathrm{E}}\right)$, and a discounting $(\gamma)$ parameter $\left(\mathrm{LR}_{-} \mathrm{W}_{\mathrm{R}_{-}} \mathrm{W}_{\mathrm{E}_{-}} \gamma\right)$; V) a

824 model with 2 learning rates $\left(\alpha_{R}, \alpha_{E}\right)$, a reward weight $\left(W_{R}\right)$, and a discounting $(\gamma)$ parameter

825 (2LR_W $\left.W_{R} \gamma\right)$; VI) a model with 2 learning rates $\left(\alpha_{R}, \alpha_{E}\right)$, a reward weight $\left(W_{R}\right)$, effort weight

$826\left(\mathrm{~W}_{\mathrm{E}}\right)$ and discounting $(\gamma)$ parameter $\left(2 \mathrm{LR}_{-} \mathrm{W}_{\mathrm{R}_{-}} \mathrm{W}_{\mathrm{E}_{-}} \gamma\right)$

827 Lower/upper bounds for all parameters were set to $[0,1]$ and all models contained a $\beta$

828 parameter. Consistent with previous work (Skvortsova et al., 2017; Skvortsova et al., 2014),

829 reward and action cost outcomes were set to [0,1 for no/yes reward] and [-1,0 for no/yes

830 effort avoidance], respectively. 


\section{Pupillometry}

832 Fluctuations in pupil diameter were continuously measured using an SR-Research Eyelink

8331000 Tower Mount infrared eye tracker while participants performed the reward

834 maximization/action cost minimization reinforcement learning task (1000Hz sampling rate,

835 except for three participants, whose data were obtained at 500Hz). Participants placed their

836 head on an adjustable chin rest and against a forehead bar to minimize motion. Eye-tracker

837 calibration was performed at the start of the paradigm, and subsequently every $10 \mathrm{~min}$.

838 Stimulus luminance was matched using the SHINE toolbox (Willenbockel et al., 2010) in

839 MATLAB (v. 2014B; The MathWorks, Inc., Natick, Massachusetts, United States). Due to

840 the COVID-19 pandemic, pupillometry data were not collected for the final eight

841 participants. Three participants, moreover, failed the quality control for eye-tracking data (2

842 no-stress control/1 acute stress) leaving a final sample of 69 participants with eye-tracking

843 data (34 no-stress control/35 acute stress).

844 Eye-tracking data were pre-processed using an open source pre-processing toolbox

845 (Kret \& Sjak-Shie, 2019) and in accordance with previous work (Jackson \& Sirois, 2009).

846 Blinks and other invalid samples, due to dilation speed, deviation from the trend line, and

847 extreme values (Kret \& Sjak-Shie, 2019) were removed, interpolated, smoothed (4Hz low-

848 pass filter, fourth-order Butterworth filter) (Jackson \& Sirois, 2009), z-scored and down-

849 sampled to $50 \mathrm{hz}$ (i.e., $20 \mathrm{~ms}$ ). Bins with fewer than $80 \%$ valid samples were removed

850 (Lawson et al., 2020). For analyses, we considered three epochs of interest: choice (-1500ms

851 pre-choice $-15000 \mathrm{~ms}$ post-choice), effort outcome (0-1000ms post-outcome), and reward

852 outcome (0-2000ms post-outcome). We reduced the duration of the effort outcome epoch to

$8531000 \mathrm{~ms}$ to minimize force exertion-related effects on pupil size (see below). Recent work has

854 shown that expectation violations (prediction errors) are encoded by pupil size fluctuations

855 within this timeframe (Lawson et al., 2020). Given that we observed large grip force- 
associated effects on the pupillometry signal (see Figure Supplement 8 middle row, for a outcome trials in Figure Supplement 8.

\section{Statistical analyses}

862 Statistical analyses were conducted using R, version 3.6.2 (Team, 2020) and, where applicable, results were visualised using Raincloud Plots (Allen, Poggiali, Whitaker, Marshall, \& Kievit, 2019). Acute stress measurements were analysed using mixed ANOVAs involving Condition (between-factor condition: no-stress control, acute stress induction) and Time (within-factor: 2 pre/post-MAST or 6 levels for sCORT). total trial amount ( $n=30$ per pair). Mixed ANOVAs involving Condition, Trial Type (RL, EL) and Difficulty (Easy, Hard pairs) were carried out. For analyses involving Time effects (i.e., repeated presentations of stimulus pairs), accuracy scores were averaged per bin of ten presentations (presentation 1-10, 11-20, and 21-30). To better understand whether acute stress effects on task performance were primarily driven by changes in sensitivity to positive or negative outcomes, win-stay (repeating a choice following a positive outcome) and loseshift (choosing the other stimulus following a loss) rates were calculated for RL and EL trials

876 (Hanneke E. M. den Ouden et al., 2013). For RL trials, we calculated win-stay/lose-shift rates 877 using reward outcomes (yes/no reward); for EL trials we used effort outcomes (yes/no effort).

878 We refer to the 2-level factor representing win-stay/lose-shift rates as "Strategy". For surprise 879 test trials involving the original four pairs ( $\mathrm{n}=4$ presentations per pair), we investigated final 880 choice tendences using a one-sample t-test against chance level (0.5). Participants' ability to 
discriminate stimuli based on reward value and action cost in novel stimulus arrangements

$882(\mathrm{n}=48,24$ reward value and 24 action cost discrimination trials) were investigated using mixed ANOVAs involving Condition and Trial Type. investigated using Condition-by learning rate $\left(\alpha_{R}, \alpha_{\mathrm{E}}\right)$ mixed ANOVAs and independent samples t-tests. Given that we used separate priors for the two groups, we report the Bayesian analogue of a t-test and mixed-ANOVA (Kruschke, 2014) - a more robust test of group differences - for posterior parameters obtained from the hierarchically fit model (for reference, we also report these analyses for the non-hierarchical data). Post hoc (simple) main effect analyses for all ANOVAs were conducted using independent sample (Condition), paired-samples (Time, Trial Type, Strategy), and onesample t-tests ( $\neq 0$ or 0.5 ). Greenhouse-Geisser-corrected statistics were reported when sphericity assumptions were violated. We report statistical significance as $p<0.05$ (twosided), but we note that most main and interaction effects involving Condition survived at a more stringent threshold $(p<.01)$, except for some strategy and surprise test phase effects, which should be interpreted with caution. In case of statistically significant results, generalized eta square $\left(\right.$ ges; $\left.\mathrm{n}^{2} \mathrm{G}\right)$ was reported, with $\mathrm{n}^{2} \mathrm{G}$ values of $0.02,0.06$, and 0.14 representing a small, medium, and large effect size, respectively (Lakens, 2013).

900 model-free analyses, we investigated group differences in pupil size during the choice, effort

901 outcome, and reward outcome stage, for every bin of interest. To better understand how putative activity of ascending neuromodulatory systems may drive stress-induced changes in computational strategies that support reward maximisation/action cost minimisation learning,

904 we conducted model-based pupillometry analyses using computational parameters from the 905 winning (2LR_ $\gamma$ ) model (Lawson et al., 2020). First, we used linear regression to estimate 
906 beta weights for the association between pupil size and computational estimates of task-

907 related behaviour for every participant, for every epoch, for every bin. For the choice phase,

908 we regressed trial-wise measures of pupil size against trial-wise estimates of the subjective

909 decision value (i.e., effort-discounted reward value) of the chosen stimulus. For the effort and

910 reward outcome phase, trial-wise EPEs and RPEs were the primary predictors of interest,

911 respectively. Trial number (1-120) and presented images/pair (RL_easy, RL_hard, EL_easy,

912 EL hard) served as additional predictors of interest for all models. Additional epoch-specific

913 variables of interest were included for the choice (optimal choice yes/no), effort (action cost

914 of chosen stimulus, effort avoidance yes/no), and reward (reward value of chosen stimulus,

915 reward yes/no, effort avoidance yes/no) outcome phase. Similar results were obtained when

916 repeating the analyses with more elaborate GLMs (e.g., the addition of yes/no most likely

917 outcome based on reward/effort outcome probabilities ["surprise"] and reward/action cost for

918 EL/RL trials). Secondly, in group-level GLMs, we compared the resulting beta weights I)

919 against zero (for the no-stress control/acute stress condition separately), to investigate when

920 the pupil encoded the computational process of interest, and II) between groups, to assess

921 stress-induced changes in associations between pupil size and computational processes. To

922 control the false positive rate, we conducted permutation tests at the bin- and cluster-level

923 (2000 permutations, $\left.\alpha_{\text {permute }}=0.05\right)$. All correlations were performed using Spearman's $\rho$

924 correlations. Permutation tests were also conducted for correlation analyses involving acute

925 stress measures and pupil encoding of predictions errors. 


\section{Figure 3 - Source Data 1}

927 Source files for task performance data.

928 This link contains all task performance data used for the analyses shown in Figure 3. Raw

929 data can be found under "task_performance".

930 https://osf.io/ydv2q/

931

932 Figure 6 - Source Data 2

933 Source files for pupillometry data.

934 This link contains pupillometry data used for the analyses shown in Figure 6. Raw data can

935 be found under "pupillometry".

936 https://osf.io/ydv2q/

937

938 Acknowledgements

939 We are indebted to Drs. Conny Quaedflieg, Edwin S. Dalmaijer. and Ross D. Markello for

940 advice on stress-induction procedures and paradigm development. We thank Dr. Michael

941 Frank for advice on hierarchical computational modelling procedures. We thank Katya Brat-

942 Matchett for involvement in participant recruitment and Truda Driessen for administrative

943 support.

944

945 Competing interests

946 D.H. has received financial compensation as a consultant for P1vital Products Ltd. These

947 activities were unrelated to the work presented in this manuscript. The authors declare no

948 competing interests. 


\section{References}

950

951

952

953

954

955

956

957

958

959

960

961

962

963

964

965

966

967

968

969

970

971

Allen, M., Poggiali, D., Whitaker, K., Marshall, T. R., \& Kievit, R. A. (2019). Raincloud plots: a multi-platform tool for robust data visualization. Wellcome Open Res, 4, 63. doi:10.12688/wellcomeopenres.15191.1

Apps, M. A. J., Grima, L. L., Manohar, S., \& Husain, M. (2015). The role of cognitive effort in subjective reward devaluation and risky decision-making. Scientific reports, 5(1), 16880. doi:10.1038/srep 16880

Arnsten, A. F. (2015). Stress weakens prefrontal networks: molecular insults to higher cognition. Nat Neurosci, 18(10), 1376-1385. doi:10.1038/nn.4087

Arnsten, A. F., \& Goldman-Rakic, P. S. (1998). Noise stress impairs prefrontal cortical cognitive function in monkeys: evidence for a hyperdopaminergic mechanism. Arch Gen Psychiatry, 55(4), 362-368. doi:10.1001/archpsyc.55.4.362

Baik, J.-H. (2020). Stress and the dopaminergic reward system. Experimental \& Molecular Medicine, 52(12), 1879-1890. doi:10.1038/s12276-020-00532-4

Bailey, S. L., \& Heitkemper, M. M. (2001). Circadian rhythmicity of cortisol and body temperature: morningness-eveningness effects. Chronobiology International, 18(2), 249-261. doi:10.1081/CBI-100103189

Berghorst, L. H., Bogdan, R., Frank, M. J., \& Pizzagalli, D. A. (2013). Acute stress selectively reduces reward sensitivity. Front Hum Neurosci, 7, 133. doi:10.3389/fnhum.2013.00133

Bryce, C. A., \& Floresco, S. B. (2016). Perturbations in Effort-Related Decision-Making Driven by Acute Stress and Corticotropin-Releasing Factor. Neuropsychopharmacology, 41(8), 2147-2159. doi:10.1038/npp.2016.15 
972

973

974

975

976

977

978

979

980

981

982

983

984

985

986

987

988

989

990

991

992

993

994

995

Byrne, K. A., Cornwall, A. C., \& Worthy, D. A. (2019). Acute stress improves long-term reward maximization in decision-making under uncertainty. Brain and Cognition, 133, 84-93. doi:https://doi.org/10.1016/j.bandc.2019.02.005

Cannon, W. B. (1915). Bodily changes in pain, hunger, fear, and rage. CHAPTER XI: The utility of the bodily changes in pain and great emotion: D. Appleton and company.

Carvalheiro, J., Conceição, V. A., Mesquita, A., \& Seara-Cardoso, A. (2020). Acute stress impairs reward learning in men. Brain Cogn, 147, 105657. doi:10.1016/j.bandc.2020.105657

Daw, N. D. (2011). Trial-by-trial data analysis using computational models. Decision making, affect, and learning: Attention and performance XXIII, 23(1).

de Berker, A. O., Tirole, M., Rutledge, R. B., Cross, G. F., Dolan, R. J., \& Bestmann, S. (2016). Acute stress selectively impairs learning to act. Scientific reports, 6(1), 29816. doi:10.1038/srep29816

de Kloet, E. R., Joëls, M., \& Holsboer, F. (2005). Stress and the brain: from adaptation to disease. Nature Reviews Neuroscience, 6(6), 463-475. doi:10.1038/nrn1683

Del Arco, A., Park, J., \& Moghaddam, B. (2020). Unanticipated Stressful and Rewarding Experiences Engage the Same Prefrontal Cortex and Ventral Tegmental Area Neuronal Populations. eneuro, 7(3), ENEURO.0029-0020.2020. doi:10.1523/ENEURO.0029-20.2020

den Ouden, H. E., Swart, J. C., Schmidt, K., Fekkes, D., Geurts, D. E., \& Cools, R. (2015). Acute serotonin depletion releases motivated inhibition of response vigour. Psychopharmacology (Berl), 232(7), 1303-1312. doi:10.1007/s00213-014-3762-4

den Ouden, Hanneke E. M., Daw, Nathaniel D., Fernandez, G., Elshout, Joris A., Rijpkema, M., Hoogman, M., .. . Cools, R. (2013). Dissociable Effects of Dopamine and 
Serotonin on Reversal Learning. Neuron, 80(4), 1090-1100.

997 doi:https://doi.org/10.1016/j.neuron.2013.08.030

998

999

1000

1001

1002

1003

1004

1005

1006

1007

1008

1009

1010

1011

1012

1013

1014

1015

1016

1017

1018

Dickerson, S. S., \& Kemeny, M. E. (2004). Acute stressors and cortisol responses: a theoretical integration and synthesis of laboratory research. Psychol Bull, 130(3), 355391. doi:10.1037/0033-2909.130.3.355

Evans, K. L., \& Hampson, E. (2015). Sex-dependent effects on tasks assessing reinforcement learning and interference inhibition. Frontiers in psychology, 6, 1044-1044. doi:10.3389/fpsyg.2015.01044

Frank, M. J., Seeberger, L. C., \& Reilly, R. C. (2004). By Carrot or by Stick: Cognitive Reinforcement Learning in Parkinsonism. science, 306(5703), 1940. doi:10.1126/science.1102941

Frey, A.-L., Frank, M. J., \& McCabe, C. (2019). Social reinforcement learning as a predictor of real-life experiences in individuals with high and low depressive symptomatology. Psychological Medicine, 1-8. doi:10.1017/S0033291719003222

Friedman, A., Homma, D., Bloem, B., Gibb, L. G., Amemori, K. I., Hu, D., . . Graybiel, A. M. (2017). Chronic Stress Alters Striosome-Circuit Dynamics, Leading to Aberrant Decision-Making. Cell, 171(5), 1191-1205.e1128. doi:10.1016/j.cell.2017.10.017

Guitart-Masip, M., Huys, Q. J. M., Fuentemilla, L., Dayan, P., Duzel, E., \& Dolan, R. J. (2012). Go and no-go learning in reward and punishment: interactions between affect and effect. Neuroimage, 62(1), 154-166. doi:10.1016/j.neuroimage.2012.04.024

Hartmann, M. N., Hager, O. M., Tobler, P. N., \& Kaiser, S. (2013). Parabolic discounting of monetary rewards by physical effort. Behavioural Processes, 100, 192-196. doi:https://doi.org/10.1016/j.beproc.2013.09.014 
1019 Hauser, T. U., Eldar, E., \& Dolan, R. J. (2017). Separate mesocortical and mesolimbic pathways encode effort and reward learning signals. Proceedings of the National Academy of Sciences, 114(35), E7395. doi:10.1073/pnas.1705643114

1022 Hermans, E. J., Henckens, M. J. A. G., Joëls, M., \& Fernández, G. (2014). Dynamic adaptation of large-scale brain networks in response to acute stressors. Trends in

Hermans, E. J., van Marle, H. J. F., Ossewaarde, L., Henckens, M. J. A. G., Qin, S., van Kesteren, M. T. R., . . . Fernández, G. (2011). Stress-Related Noradrenergic Activity

Hernandez Lallement, J., Kuss, K., Trautner, P., Weber, B., Falk, A., \& Fliessbach, K. (2014). Effort increases sensitivity to reward and loss magnitude in the human brain. Social cognitive and affective neuroscience, 9(3), 342-349. doi:10.1093/scan/nss147

Hernaus, D., Frank, M. J., Brown, E. C., Brown, J. K., Gold, J. M., \& Waltz, J. A. (2019). Impaired Expected Value Computations in Schizophrenia Are Associated With a Reduced Ability to Integrate Reward Probability and Magnitude of Recent Outcomes. Biol Psychiatry Cogn Neurosci Neuroimaging, 4(3), 280-290. doi:10.1016/j.bpsc.2018.11.011

Hernaus, D., Gold, J. M., Waltz, J. A., \& Frank, M. J. (2018). Impaired Expected Value Computations Coupled With Overreliance on Stimulus-Response Learning in Schizophrenia. Biol Psychiatry Cogn Neurosci Neuroimaging, 3(11), 916-926. doi:10.1016/j.bpsc.2018.03.014

Hernaus, D., Quaedflieg, C. W., Offermann, J. S., Casales Santa, M. M., \& van Amelsvoort, T. (2018). Neuroendocrine stress responses predict catecholamine-dependent working 
memory-related dorsolateral prefrontal cortex activity. Social cognitive and affective neuroscience, 13(1), 114-123.

1045 Huys, Q. J., Pizzagalli, D. A., Bogdan, R., \& Dayan, P. (2013). Mapping anhedonia onto reinforcement learning: a behavioural meta-analysis. Biology of mood \& anxiety

Inzlicht, M., Shenhav, A., \& Olivola, C. Y. (2018). The Effort Paradox: Effort Is Both Costly and Valued. Trends in cognitive sciences, 22(4), 337-349. doi:10.1016/j.tics.2018.01.007

Jackson, I., \& Sirois, S. (2009). Infant cognition: going full factorial with pupil dilation. Dev Sci, 12(4), 670-679. doi:10.1111/j.1467-7687.2008.00805.x

Joëls, M., Pu, Z., Wiegert, O., Oitzl, M. S., \& Krugers, H. J. (2006). Learning under stress: how does it work? Trends in cognitive sciences, 10(4), 152-158. doi:https://doi.org/10.1016/j.tics.2006.02.002

Joshi, S., Li, Y., Kalwani, R. M., \& Gold, J. I. (2016). Relationships between Pupil Diameter and Neuronal Activity in the Locus Coeruleus, Colliculi, and Cingulate Cortex. Neuron, 89(1), 221-234. doi:10.1016/j.neuron.2015.11.028

Klein-Flügge, M. C., Kennerley, S. W., Friston, K., \& Bestmann, S. (2016). Neural signatures of value comparison in human cingulate cortex during decisions requiring

1062 Koh, D., Ng, V., \& Naing, L. (2014). Alpha Amylase as a Salivary Biomarker of Acute Stress of Venepuncture from Periodic Medical Examinations. Frontiers in Public Health, 2(121). doi:10.3389/fpubh.2014.00121

Kret, M. E., \& Sjak-Shie, E. E. (2019). Preprocessing pupil size data: Guidelines and code. Behav Res Methods, 51(3), 1336-1342. doi:10.3758/s13428-018-1075-y

1067 Kruschke, J. (2014). Doing Bayesian data analysis: A tutorial with R, JAGS, and Stan. 
Lakens, D. (2013). Calculating and reporting effect sizes to facilitate cumulative science: a practical primer for t-tests and ANOVAs. Frontiers in psychology, 4, 863-863. doi:10.3389/fpsyg.2013.00863

Lawson, R. P., Bisby, J., Nord, C. L., Burgess, N., \& Rees, G. (2020). The Computational, Pharmacological, and Physiological Determinants of Sensory Learning under Uncertainty. Current Biology. doi:https://doi.org/10.1016/j.cub.2020.10.043

Le Heron, C., Plant, O., Manohar, S., Ang, Y. S., Jackson, M., Lennox, G., . . Husain, M. (2018). Distinct effects of apathy and dopamine on effort-based decision-making in Parkinson's disease. Brain, 141(5), 1455-1469. doi:10.1093/brain/awy110

Lefebvre, G., Lebreton, M., Meyniel, F., Bourgeois-Gironde, S., \& Palminteri, S. (2017). Behavioural and neural characterization of optimistic reinforcement learning. Nature Human Behaviour, 1(4), 0067. doi:10.1038/s41562-017-0067 modulates reinforcement learning in younger and older adults. Psychology and aging, 28(1), 35-46. doi:10.1037/a0029823

Meyniel, F., Goodwin, G. M., Deakin, J. W., Klinge, C., MacFadyen, C., Milligan, H., .. . Gaillard, R. (2016). A specific role for serotonin in overcoming effort cost. Elife, 5,

Morris, B. H., \& Rottenberg, J. (2015). Heightened reward learning under stress in generalized anxiety disorder: A predictor of depression resistance? Journal of abnormal psychology, 124(1), 115.

Myung, J. I., Tang, Y., \& Pitt, M. A. (2009). Evaluation and comparison of computational models. Methods Enzymol, 454, 287-304. doi:10.1016/s0076-6879(08)03811-1 
Nassar, M. R., \& Frank, M. J. (2016). Taming the beast: extracting generalizable knowledge from computational models of cognition. Current opinion in behavioral sciences, 11, 49-54. doi:10.1016/j.cobeha.2016.04.003

Nater, U. M., Rohleder, N., Gaab, J., Berger, S., Jud, A., Kirschbaum, C., \& Ehlert, U. (2005). Human salivary alpha-amylase reactivity in a psychosocial stress paradigm. Int J Psychophysiol, 55(3), 333-342. doi:10.1016/j.ijpsycho.2004.09.009

Niv, Y. (2009). Reinforcement learning in the brain. Journal of Mathematical Psychology, 53(3), 139-154. doi:https://doi.org/10.1016/j.jmp.2008.12.005

1099 Otto, A. R., Raio, C. M., Chiang, A., Phelps, E. A., \& Daw, N. D. (2013). Working-memory capacity protects model-based learning from stress. Proceedings of the National Academy of Sciences, 110(52), 20941. doi:10.1073/pnas.1312011110

Palminteri, S., \& Pessiglione, M. (2017). Chapter 23 - Opponent Brain Systems for Reward and Punishment Learning: Causal Evidence From Drug and Lesion Studies in

Peirce, J., Gray, J. R., Simpson, S., MacAskill, M., Höchenberger, R., Sogo, H., . . Lindeløv, J. K. (2019). PsychoPy2: Experiments in behavior made easy. Behavior Research Methods, 51(1), 195-203. doi:10.3758/s13428-018-01193-y

1109 Pessiglione, M., Vinckier, F., Bouret, S., Daunizeau, J., \& Le Bouc, R. (2017). Why not try harder? Computational approach to motivation deficits in neuro-psychiatric diseases.

1112 Petzold, A., Plessow, F., Goschke, T., \& Kirschbaum, C. (2010). Stress reduces use of negative feedback in a feedback-based learning task. Behav Neurosci, 124(2), 248255. doi:10.1037/a0018930 
1115 Pool, E., Brosch, T., Delplanque, S., \& Sander, D. (2015). Stress increases cue-triggered "wanting" for sweet reward in humans. Journal of experimental psychology. Animal learning and cognition, 41 2, 128-136.

1118 Pruessner, J. C., Kirschbaum, C., Meinlschmid, G., \& Hellhammer, D. H. (2003). Two

1119 formulas for computation of the area under the curve represent measures of total 1120 hormone concentration versus time-dependent change. Psychoneuroendocrinology, 28(7), 916-931. doi:10.1016/s0306-4530(02)00108-7

1122 Qin, S., Hermans, E. J., van Marle, H. J. F., Luo, J., \& Fernández, G. (2009). Acute Psychological Stress Reduces Working Memory-Related Activity in the Dorsolateral Prefrontal Cortex. Biological psychiatry, 66(1), 25-32. doi:https://doi.org/10.1016/j.biopsych.2009.03.006

Raio, C. M., Hartley, C. A., Orederu, T. A., Li, J., \& Phelps, E. A. (2017). Stress attenuates

Rescorla, R. A. (1972). A theory of Pavlovian conditioning: Variations in the effectiveness of reinforcement and nonreinforcement. Current research and theory, 64-99.

1132 Rigoux, L., Stephan, K. E., Friston, K. J., \& Daunizeau, J. (2014). Bayesian model selection for group studies - revisited. Neuroimage, 84, 971-985.

1135 Russell, G., \& Lightman, S. (2019). The human stress response. Nat Rev Endocrinol, 15(9), 525-534. doi:10.1038/s41574-019-0228-0

1137 Schmidt, L., Lebreton, M., Cléry-Melin, M. L., Daunizeau, J., \& Pessiglione, M. (2012). 1138 Neural mechanisms underlying motivation of mental versus physical effort. PLoS 1139 Biol, 10(2), e1001266. doi:10.1371/journal.pbio.1001266 
1140 Schoofs, D., Wolf, O. T., \& Smeets, T. (2009). Cold pressor stress impairs performance on working memory tasks requiring executive functions in healthy young men. Behav Neurosci, 123(5), 1066-1075. doi:10.1037/a0016980

1143 Schubert, C., Lambertz, M., Nelesen, R. A., Bardwell, W., Choi, J. B., \& Dimsdale, J. E. 1144 (2009). Effects of stress on heart rate complexity--a comparison between short-term and chronic stress. Biological psychology, 80(3), 325-332. doi:10.1016/j.biopsycho.2008.11.005

1147 Schultz, W., Dayan, P., \& Montague, P. R. (1997). A neural substrate of prediction and reward. science, 275(5306), 1593-1599. doi:10.1126/science.275.5306.1593

1149 Schwabe, L., Dickinson, A., \& Wolf, O. T. (2011). Stress, habits, and drug addiction: a psychoneuroendocrinological perspective. Exp Clin Psychopharmacol, 19(1), 53-63. doi:10.1037/a0022212

Schwabe, L., \& Wolf, O. T. (2011). Stress-induced modulation of instrumental behavior: from goal-directed to habitual control of action. Behavioural brain research, 219(2), $321-328$.

Shafiei, N., Gray, M., Viau, V., \& Floresco, S. B. (2012). Acute stress induces selective alterations in cost/benefit decision-making. Neuropsychopharmacology, 37(10), 21942209. doi:10.1038/npp.2012.69 
1165 Smeets, T., Cornelisse, S., Quaedflieg, C. W., Meyer, T., Jelicic, M., \& Merckelbach, H.

1166

1167

1168

1169

1170

1171

1172

1173

1174

1175

1176

1177

1178

1179

1180

1181

1182

1183

1184

1185

1186

1187

1188

1189

(2012). Introducing the Maastricht Acute Stress Test (MAST): a quick and noninvasive approach to elicit robust autonomic and glucocorticoid stress responses. Psychoneuroendocrinology, 37(12), 1998-2008. doi:10.1016/j.psyneuen.2012.04.012

Steinberg, E. E., Keiflin, R., Boivin, J. R., Witten, I. B., Deisseroth, K., \& Janak, P. H. (2013). A causal link between prediction errors, dopamine neurons and learning. Nature neuroscience, 16(7), 966-973. doi:10.1038/nn.3413

Stelly, C. E., Tritley, S. C., Rafati, Y., \& Wanat, M. J. (2020). Acute Stress Enhances Associative Learning via Dopamine Signaling in the Ventral Lateral Striatum. The Journal of Neuroscience, 40(22), 4391. doi:10.1523/JNEUROSCI.3003-19.2020

Team, R. C. (2020). R: A language and environment for statistical computing. R Foundation for Statistical Computing. Retrieved from https://www.Rproject.org/

Timmers, I., Kaas, A. L., Quaedflieg, C., Biggs, E. E., Smeets, T., \& de Jong, J. R. (2018). Fear of pain and cortisol reactivity predict the strength of stress-induced hypoalgesia. Eur J Pain, 22(7), 1291-1303. doi:10.1002/ejp.1217

Valton, V., Wise, T., \& Robinson, O. J. (2020). Recommendations for Bayesian hierarchical model specifications for case-control studies in mental health. arXiv preprint arXiv:2011.01725.

Varazzani, C., San-Galli, A., Gilardeau, S., \& Bouret, S. (2015). Noradrenaline and dopamine neurons in the reward/effort trade-off: a direct electrophysiological comparison in behaving monkeys. J Neurosci, 35(20), 7866-7877. doi:10.1523/jneurosci.0454-15.2015

Watkins, C. J. C. H., \& Dayan, P. (1992). Q-learning. Machine Learning, 8(3), 279-292. doi:10.1007/BF00992698 
1190 Watson, D., Clark, L. A., \& Tellegen, A. (1988). Development and validation of brief measures of positive and negative affect: the PANAS scales. J Pers Soc Psychol, 54(6), 1063-1070. doi:10.1037//0022-3514.54.6.1063

1193 Willenbockel, V., Sadr, J., Fiset, D., Horne, G. O., Gosselin, F., \& Tanaka, J. W. (2010).

1194 Controlling low-level image properties: The SHINE toolbox. Behavior Research Methods, 42(3), 671-684. doi:10.3758/BRM.42.3.671

1196 Wilson, R. C., \& Collins, A. G. (2019). Ten simple rules for the computational modeling of behavioral data. Elife, 8, e49547.

1198 Wright, B. J., O'Brien, S., Hazi, A., \& Kent, S. (2014). Increased systolic blood pressure 1199 reactivity to acute stress is related with better self-reported health. Scientific reports, 4, 6882-6882. doi:10.1038/srep06882

1201 Yohn, S. E., Errante, E. E., Rosenbloom-Snow, A., Somerville, M., Rowland, M., Tokarski, K., ... Salamone, J. D. (2016). Blockade of uptake for dopamine, but not norepinephrine or 5-HT, increases selection of high effort instrumental activity: Implications for treatment of effort-related motivational symptoms in psychopathology. Neuropharmacology, 109, 270-280. doi:10.1016/j.neuropharm.2016.06.018 


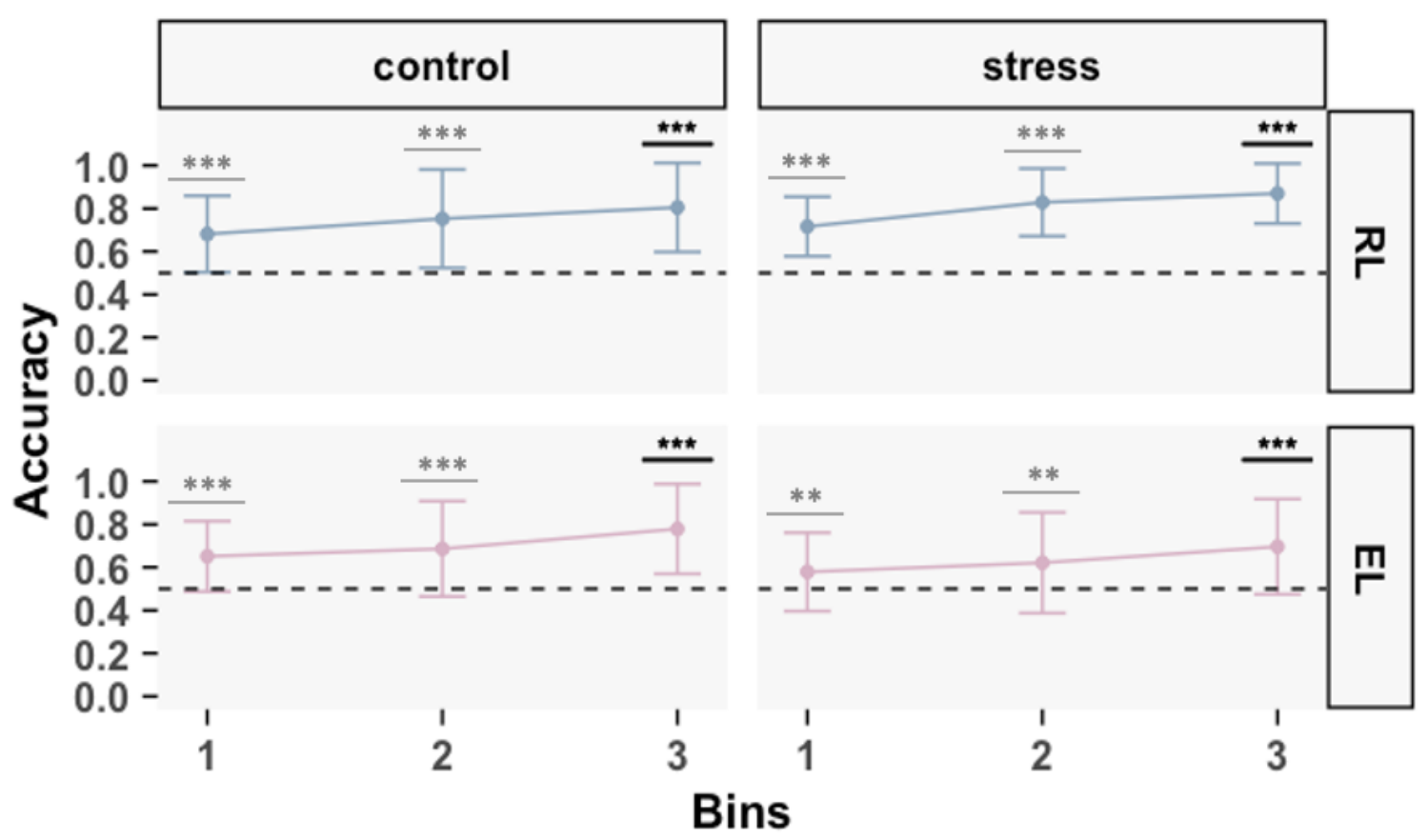

1211 Evidence of reward and action cost reinforcement learning.

1212 Optimal stimulus choices (“accuracy") on reward learning (RL) and effort learning (EL)

1213 (rows) trials for both conditions (columns). Trials were binned into groups of 10

1214 presentations. Participants performed significantly better than chance level in all bins. Means

$1215 \pm$ SD. Significant differences are denoted by asterisks $(*: p<0.05, * *: p<0.01, * * *: p<$

$12160.001)$ 


\section{Figure Supplement 2}

CONTROL

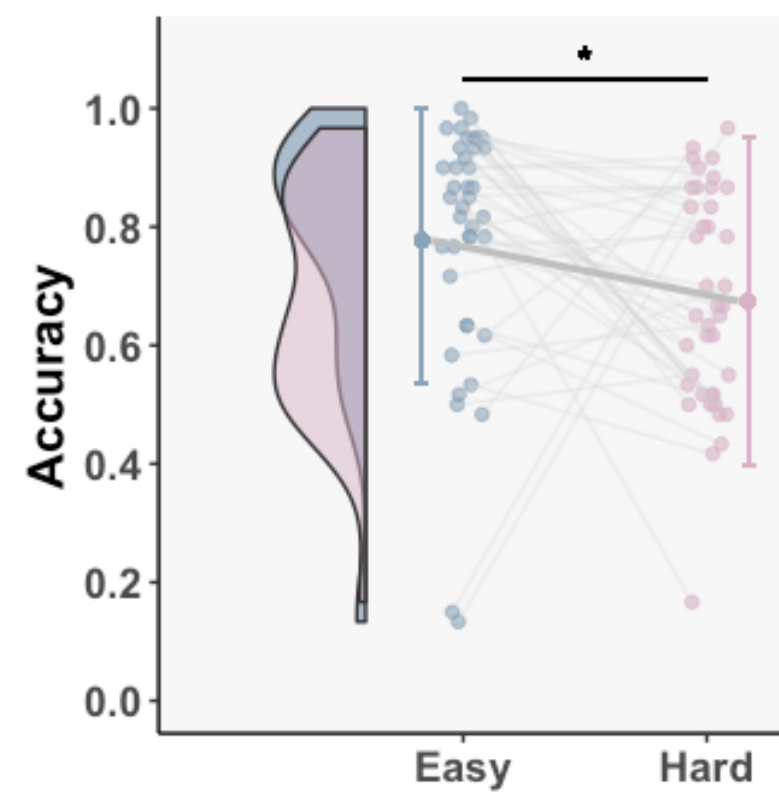

STRESS

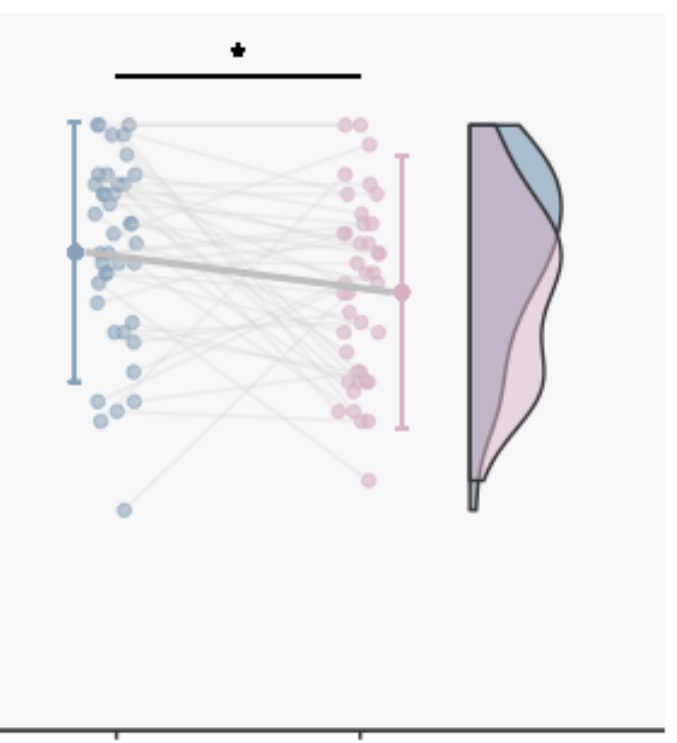

Easy

Hard

1219 Acute stress does not affect difficulty learning.

1220 Easy and hard pairs collapsed across RL/EL trials depicted for each condition separately.

1221 While all participants sampled the optimal choices more frequently for Easy vs. Hard pairs,

1222 no significant Condition-by-Difficulty interaction or between-group differences were

1223 observed. Means $\pm \mathrm{SD}$, individual data points, distribution and density of the data are

1224 displayed. Significant differences are denoted by asterisks $(*: p<0.05, * *: p<0.01, * * *: p<$ $12250.001)$. 
bioRxiv preprint doi: https://doi.org/10.1101/2021.04 25.441347; this version posted April 27, 2021. The copyright holder for this preprint (which was not certified by peer review) is the author/funder, who has granted bioRxiv a license to display the preprint in perpetuity. It is made available under aCC-BY 4.0 International license.

CONTROL

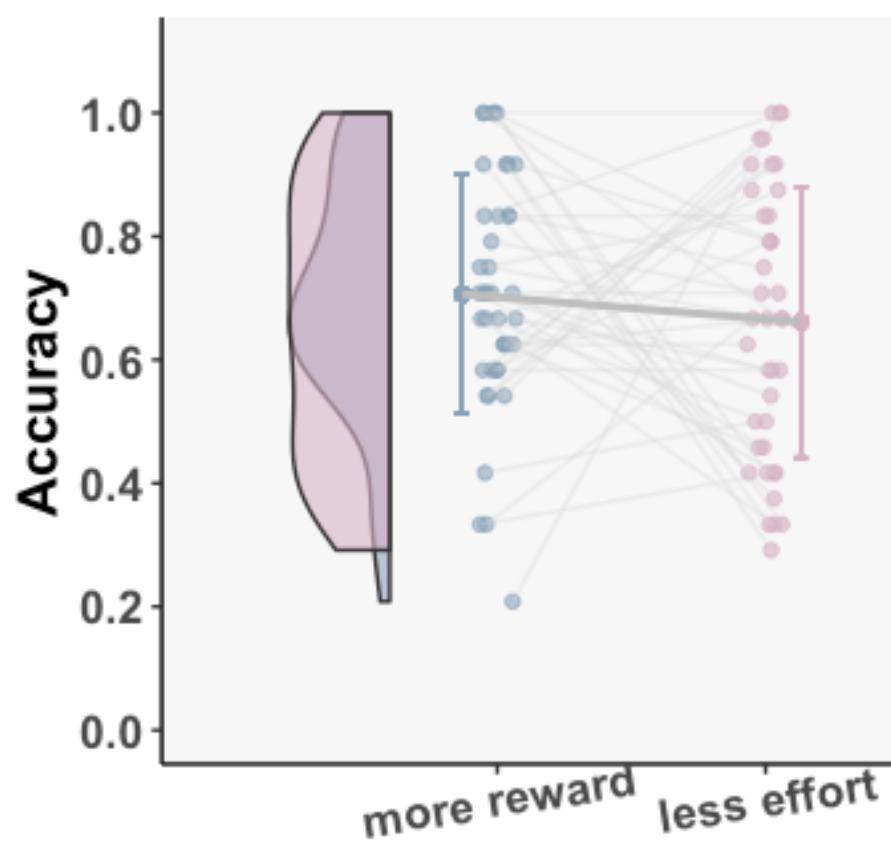

STRESS

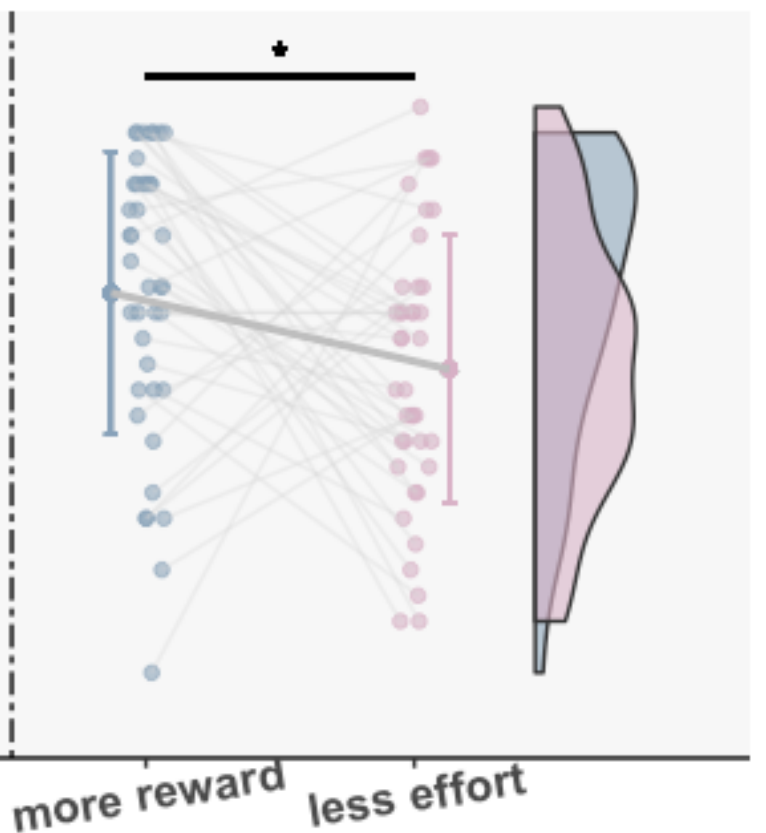

1228 Surprise test phase performance.

1229 The acute stress group performed better on reward than action cost discrimination trials.

1230 Means $\pm \mathrm{SD}$, individual data points, distribution and density of the data are displayed.

1231 Significant differences are depicted with asterisks $(*: p<0.05, * *: p<0.01, * * *: p<0.001)$. 


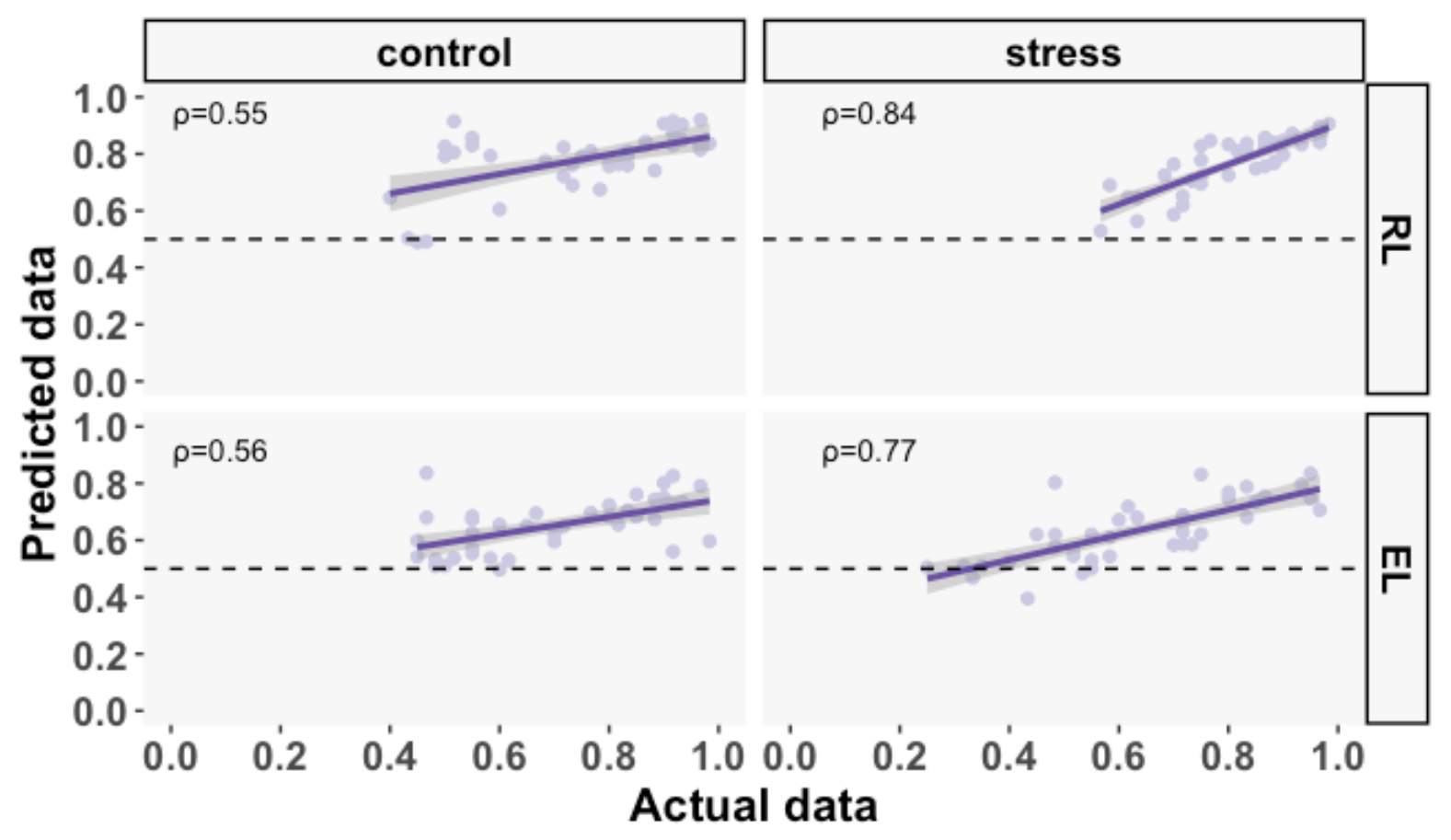
Actual data

1234 Correlations between empirical and simulated 2LR_ $\gamma$ choices.

1235 Actual and post hoc simulated choices for RL and EL (rows) were highly correlated both for

1236 no-stress control and acute stress subjects (columns). Simulations were averaged across 10

1237 repetitions per subject. Solid and shaded lines represent mean $\pm \mathrm{C} \mathrm{I}_{95 \%}$. Dots represent

1238 individual data points. Horizontal dashed lines indicate chance level (0.5). 

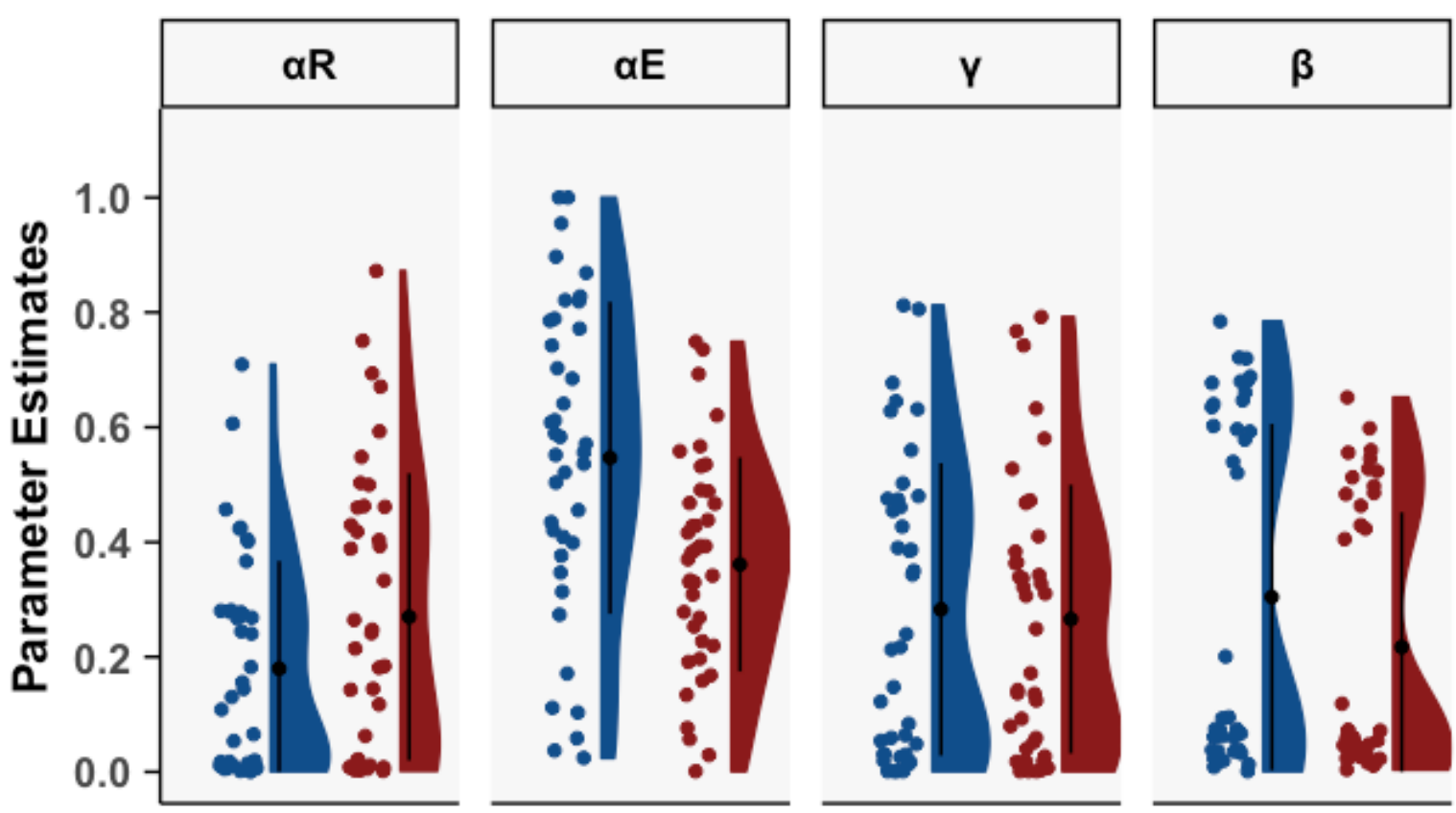

Parameter estimates after Bayesian hierarchical model fitting.

1242 Hierarchical model fitting reproduced the overall pattern of parameter estimates (Figure 5 for 1243 comparison) . 


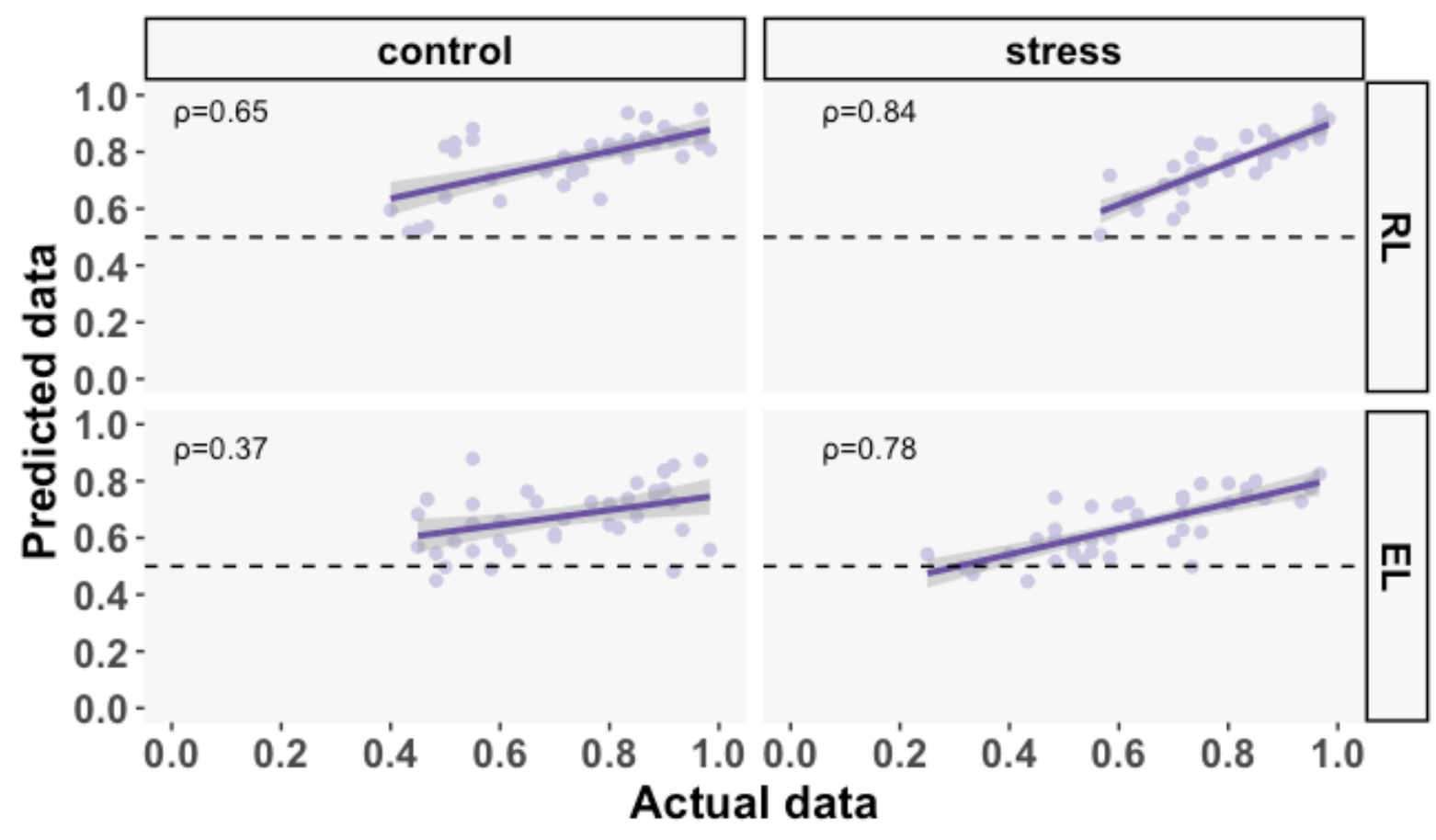

1246 Correlations between empirical and simulated 2LR_ $\gamma$ choices after Bayesian

1247 hierarchical model fitting.

1248 Correlations between actual and post hoc simulated choices for RL and EL (rows) for no-

1249 stress control and acute stress subjects (columns). Simulations were averaged across 10

1250 repetitions per subject. Solid and shaded lines represent mean $\pm \mathrm{CI} 95 \%$. Dots represent

1251 individual data points. Horizontal dashed lines indicate chance level (0.5). 


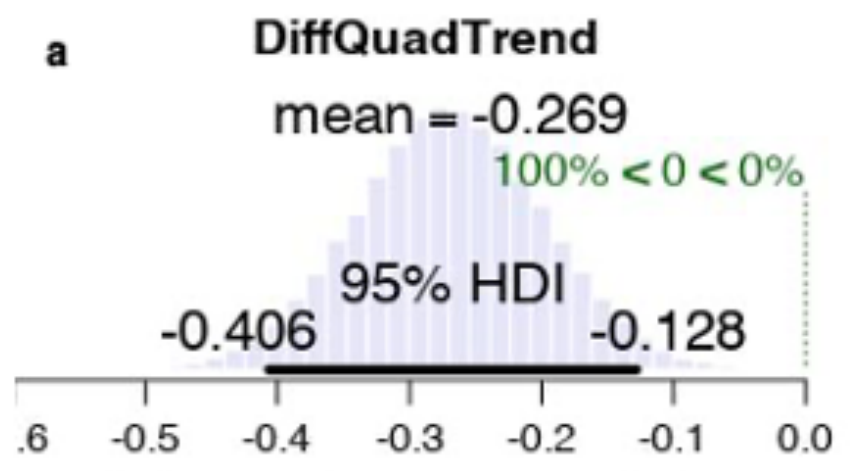

A1B1vA2B1

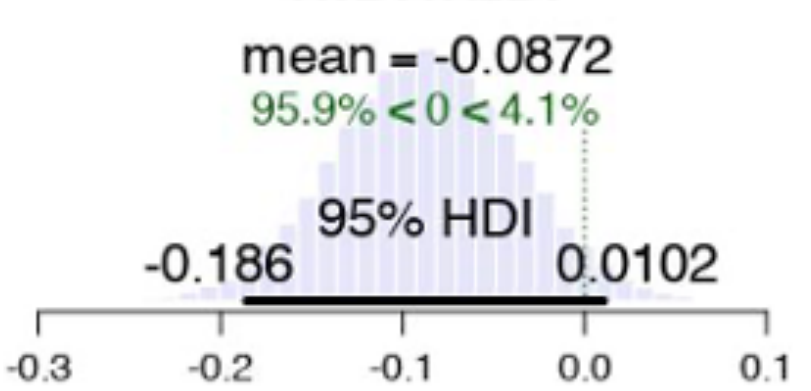

c

A1B1vA2B1

mean $=0.183$

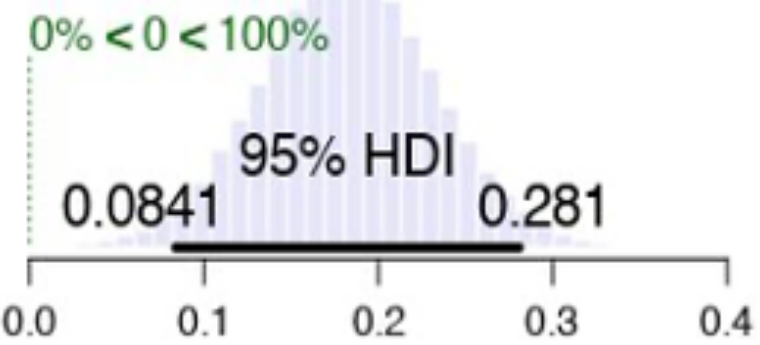

1254 Bayesian estimation analysis to evaluate group differences in posterior parameter

\section{5 distributions}

1256 Panel A. Bayesian estimation (mixed-ANOVA) using posterior parameters (following

1257 hierarchical fitting) revealed evidence for a credible Condition-by-Learning Rate interaction.

1258 The observed mean difference from zero that falls outside the 95\% HDI suggests that the 
bioRxiv preprint doi: https://doi.org/10.1101/2021.04.25.441347; this version posted April 27, 2021. The copyright holder for this preprint (which was not certified by peer review) is the author/funder, who has granted bioRxiv a license to display the preprint in perpetuity. It is made available under aCC-BY 4.0 International license.

1259 difference between $\alpha_{\mathrm{E}}$ and $\alpha_{\mathrm{R}}$ was greater in no-stress controls compared to acute stress

1260 subjects. Panel B. Both groups did not differ in the magnitude of $\alpha_{\mathrm{R}}$, as indicated by a $95 \%$

1261 HDI that included 0. Panel C. Acute stress compared to no-stress control subjects exhibited a

1262 lower value of $\alpha_{\mathrm{E}}$, as indicated by a 95\% HDI that falls well above zero. 


\section{Effort Outcome (all trials)}

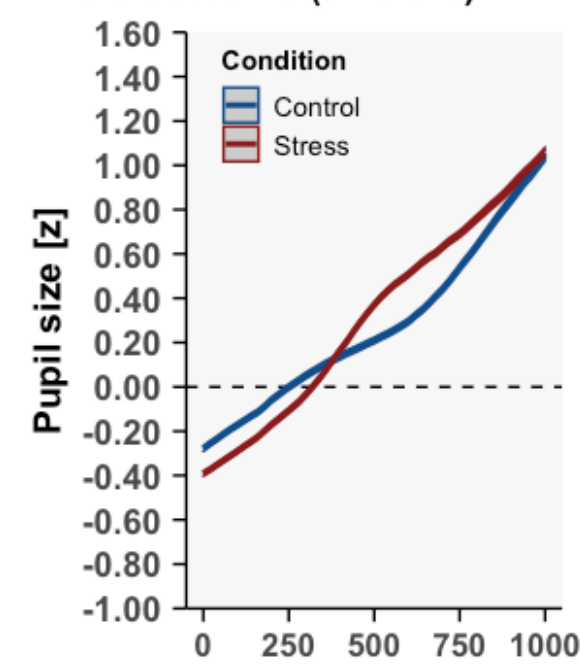
trials.
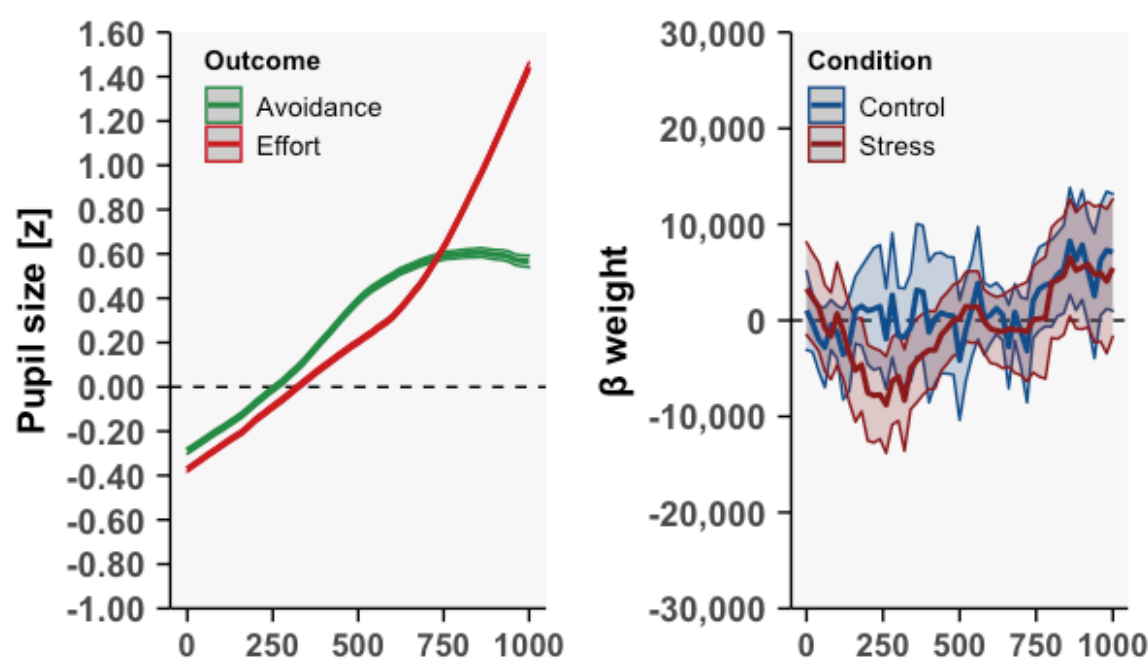

\section{Time [ms]}

\section{Pupillometry analyses using all effort outcome trials.}

Left: Model-free analyses of pupil size using all effort outcome trials. Middle: Pupil size differences during effort/effort avoidance outcomes in the entire sample; force exertion was associated with large effects on pupil size and these trials were therefore excluded from analysis. Right: Model-based action cost prediction error analyses using all effort outcome 